\title{
In vivo imaging reveals PKA regulation of ERK activity during neutrophil recruitment to inflamed intestines.
}

\section{$\operatorname{AUTHOR}(S)$ :}

Mizuno, Rei; Kamioka, Yuji; Kabashima, Kenji; Imajo, Masamichi; Sumiyama, Kenta; Nakasho, Eiji; Ito, Takeshi; ... Sakai, Yoshiharu; Kiyokawa, Etsuko; Matsuda, Michiyuki

\section{CITATION:}

Mizuno, Rei ...[et al]. In vivo imaging reveals PKA regulation of ERK activity during neutrophil recruitment to inflamed intestines.. The Journal of experimental medicine 2014, 211(6): 1123-1136

\section{ISSUE DATE:}

2014-05-19

\section{URL:}

http://hdl.handle.net/2433/187372

\section{RIGHT:}

(c) 2014 Mizuno et al.; This article is distributed under the terms of an Attribution-Noncommercial-Share Alike-No Mirror Sites license for the first six months after the publication date (see http://www.rupress.org/terms). After six months it is available under a Creative Commons License (Attribution-Noncommercial-Share Alike 3.0 Unported license, as described at http://creativecommons.org/licenses/by-nc-sa/3.0/). 


\title{
In vivo imaging reveals PKA regulation of ERK activity during neutrophil recruitment to inflamed intestines
}

\author{
Rei Mizuno, ${ }^{1,2}$ Yuji Kamioka, ${ }^{1,5}$ Kenji Kabashima, ${ }^{3}$ Masamichi Imajo, ${ }^{6}$ \\ Kenta Sumiyama, ${ }^{7}$ Eiji Nakasho,${ }^{8}$ Takeshi Ito, ${ }^{4}$ Yoko Hamazaki, ${ }^{4}$ \\ Yoshihisa Okuchi, ${ }^{1,2}$ Yoshiharu Sakai, ${ }^{2}$ Etsuko Kiyokawa, ${ }^{9}$ \\ and Michiyuki Matsuda ${ }^{1,6}$
}

\begin{abstract}
'Department of Pathology and Biology of Diseases, ${ }^{2}$ Department of Gastrointestinal Surgery, ${ }^{3}$ Department of Dermatology, and ${ }^{4}$ Department of Immunology and Cell Biology, Graduate School of Medicine; ${ }^{5}$ Innovative Techno-Hub for Integrated Medical Bio-Imaging; and ${ }^{6}$ Laboratory of Bioimaging and Cell Signaling, Department of Molecular and System Biology, Graduate School of Biostudies; Kyoto University, Kyoto 606-8501, Japan

${ }^{7}$ Division of Population Genetics, National Institute of Genetics, Mishima, Shizuoka 411-8540, Japan

${ }^{8}$ Life \& Industrial Products Development Department 1, R\&D Division, Olympus Corporation, Hachioji-shi, Tokyo 192-8507, Japan ${ }^{9}$ Department of Oncologic Pathology, Kanazawa Medical University, Kanazawa, Ishikawa 920-0293, Japan
\end{abstract}

Many chemical mediators regulate neutrophil recruitment to inflammatory sites. Although the actions of each chemical mediator have been demonstrated with neutrophils in vitro, how such chemical mediators act cooperatively or counteractively in vivo remains largely unknown. Here, by in vivo two-photon excitation microscopy with transgenic mice expressing biosensors based on Förster resonance energy transfer, we time-lapse-imaged the activities of extracellular signal-regulated kinase (ERK) and protein kinase A (PKA) in neutrophils in inflamed intestinal tissue. ERK activity in neutrophils rapidly increased during spreading on the endothelial cells and showed positive correlation with the migration velocity on endothelial cells or in interstitial tissue. Meanwhile, in the neutrophils migrating in the interstitial tissue, high PKA activity correlated negatively with migration velocity. In contradiction to previous in vitro studies that showed ERK activation by prostaglandin $E_{2}\left(P E_{2}\right)$ engagement with prostaglandin receptor EP4, intravenous administration of EP4 agonist activated PKA, inhibited ERK, and suppressed migration of neutrophils. The opposite results were obtained using nonsteroidal antiinflammatory drugs (NSAIDs). Therefore, NSAID-induced enteritis may be caused at least partially by the inhibition of EP4 receptor signaling of neutrophils. Our results demonstrate that ERK positively regulates the neutrophil recruitment cascade by promoting adhesion and migration steps.

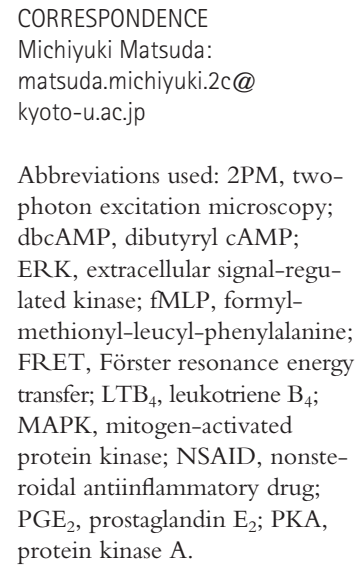

Abbreviations used: 2PM, twophoton excitation microscopy; dbcAMP, dibutyryl cAMP; ERK, extracellular signal-regulated kinase; fMLP, formylmethionyl-leucyl-phenylalanine; FRET, Förster resonance energy transfer; $\mathrm{LTB}_{4}$, leukotriene $\mathrm{B}_{4}$; MAPK, mitogen-activated protein kinase; NSAID, nonsteroidal antiinflammatory drug; $\mathrm{PGE}_{2}$, prostaglandin $\mathrm{E}_{2}$; PKA, protein kinase A.

Once inflammation occurs in tissues, in response to the gradient of chemoattractants such as leukotriene $\mathrm{B}_{4}\left(\mathrm{LTB}_{4}\right)$, IL-8, and formyl-methionylleucyl-phenylalanine (fMLP), neutrophils begin to emigrate from inside the venules to the inflammatory sites (Phillipson and Kubes, 2011). Using various in vivo microscopy approaches, it has been demonstrated that the neutrophil recruitment involves four steps: rolling, adhesion, crawling, and transmigration (Borregaard, 2010; Megens et al., 2011; Germain et al., 2012; Sanz and Kubes, 2012; Kolaczkowska and Kubes,

K. Sumiyama's present address is Cell Design Research Core Laboratory for Mouse Genetic Engineering, RIKEN Quantitative Biology Center, Chuo-ku, Kobe 650-0047, Japan.
2013).Thereafter, neutrophils that have emigrated into the interstitial tissue migrate toward the inflammatory sites by the gradient of chemoattractants. Most chemoattractant receptors expressed on neutrophils are coupled with the heterotrimeric $G_{i}$ protein, which inhibits protein kinase A (PKA) and activates p42/44 extracellular signal-regulated kinase (ERK) through both the $\alpha$ and $\beta \gamma$ subunits of $G_{i}$ (Alblas et al., 1993; Howe and Marshall, 1993; Winitz et al., 1993).

O 2014 Mizuno et al. This article is distributed under the terms of an AttributionNoncommercial-Share Alike-No Mirror Sites license for the first six months after the publication date (see http://www.rupress.org/terms). After six months it is available under a Creative Commons License (Attribution-NoncommercialShare Alike 3.0 Unported license, as described at http://creativecommons.org/ licenses/by-nc-sa/3.0/. 
The $\mathrm{G}_{\mathrm{i}}$-mediated ERK activation is required for adhesion and migration of neutrophils upon the engagement of the chemoattractants with the cognate receptors (Pillinger et al., 1996; Zarbock et al., 2007). However, this model was recently challenged by Liu et al. (2012), who proposed that AMLP-stimulated neutrophil migration is regulated negatively by ERK.

Prostaglandins at the inflammatory sites play pleiotropic roles in inflammation (Hata and Breyer, 2004; Narumiya, 2009). For example, prostaglandin $\mathrm{E}_{2}\left(\mathrm{PGE}_{2}\right)$, which is a major cyclooxygenase product in several physiological settings, regulates multiple functions of different immune cells (Ricciotti and FitzGerald, 2011; Kalinski, 2012). The main signal transduction of the four $\mathrm{PGE}_{2}$-sensitive (EP) receptors, EP1 to $\mathrm{EP} 4$, consists of a rise in intracellular cAMP concentration and subsequent PKA activation via $G_{s}$ in EP2 and EP4, a rise in intracellular free calcium ion concentration in EP1, and a decrease in intracellular cAMP concentration and ERK activation via $G_{i}$ in EP3 (Narumiya et al., 1999). Further complexity arises from the strength of the coupling to $G_{s}$ and sensitivity to the metabolic inactivation: Although both EP2 and $\mathrm{EP} 4$ receptors couple to $\mathrm{G}_{\mathrm{s}}$, the EP2 receptor transduces signals primarily through PKA, whereas the EP4 receptor primarily utilizes phosphatidylinositol 3-kinase (PI3K) and ERK (Fujino et al., 2003). EP4 signaling is rapidly desensitized after its $\mathrm{PGE}_{2}$ interaction, whereas EP2 is resistant to ligandinduced desensitization (Nishigaki et al., 1996). Reflecting these differences in molecular properties, EP2 and EP4 are regarded as pro- and antiinflammatory receptors, respectively (Kabashima et al., 2002; Hata and Breyer, 2004).

Upon activation of $\mathrm{G}_{\mathrm{s}}$-coupled receptors in many cell types, PKA suppresses ERK mitogen-activated protein kinase (MAPK) via phosphorylation and inhibition of c-Raf, a MAPK kinase (Häfner et al., 1994; Pillinger et al., 1996). In neutrophils, for example, PKA has been shown to suppress respiratory burst by inhibition of the ERK signaling (Bengis-Garber and Gruener, 1996). However, in neuronal cells, an increase in cytoplasmic cAMP can activate ERK in a Rap1-dependent manner (Vossler et al., 1997). Therefore, the regulation of ERK activity by $\mathrm{G}_{\mathrm{s}}$-coupled receptors is dependent on the cell context.

In the inflammatory tissues, neutrophils perceive several extracellular signals, which activate or inactivate ERK and PKA. Under this circumstance, it is hardly predictable which signaling pathway will be dominant in neutrophils during the course of inflammation. Although the recent advent of in vivo microscopy has enabled us to visualize the neutrophil recruitment to inflammatory sites (Megens et al., 2011; Germain et al., 2012; Sanz and Kubes, 2012), the activity change of signaling molecules has not been examined because of technical constraints. To overcome this problem, we generated transgenic mice expressing functional Förster resonance energy transfer (FRET) biosensors for ERK and showed that ERK activity correlated with migration velocity in the neutrophils of inflamed subcutaneous tissue (Kamioka et al., 2012). However, we failed to observe extravasation of neutrophils or activity change of PKA because of technical difficulty. Here, in vivo observation of the intestines of the transgenic mice enabled us to examine the role of ERK and PKA in the neutrophil recruitment to the inflammatory sites by twophoton excitation microscopy (2PM). In contradiction to a previous study that showed activation of ERK by EP4 engagement to $\mathrm{PGE}_{2}$ (Fujino et al., 2003), we show that $\mathrm{PGE}_{2}$ stimulates PKA in an EP4-dependent manner and suppresses neutrophil migration via down-regulation of ERK in the inflamed intestine.

\section{RESULTS}

\section{ERK activity during the neutrophil recruitment cascade}

We attempted to visualize ERK activity during the neutrophil recruitment cascade in the small intestines of mice after treating the intestinal tissue with LPS and AMLP to induce acute inflammation. For this purpose, we used Eisuke mice (C57BL/6-Tg(pT2A-3903NES)), which are transgenic mice expressing a cytoplasmic FRET biosensor for ERK and respond to LPS very similarly to C57BL/6 mice (not depicted). In Eisuke mice, the ERK activity can be represented by FRET/CFP ratio images (Fig. 1 A). Postcapillary venules in the lamina propria of the inflamed small intestine were liveimaged under an inverted two-photon excitation microscope. Neutrophils in the inflamed tissue could be easily distinguished by the segmented nuclei from lymphocytes or macrophages. Indeed, the cells with segmented nuclei could be stained with a neutrophil marker, anti-Gr1 antibody (not depicted). During the course of $2-5$ h of live imaging (Video 1 ), neutrophils were observed to roll on, adhere to, crawl over, and transmigrate through the endothelial cells and then to migrate between the crypts (Fig. 1, A-E; and Video 1). Timelapse videos with a shorter interval captured the precise timing of ERK activation during the neutrophil recruitment cascade (Fig. 1 C). At the beginning of the adhesion step when neutrophils arrested on the endothelial cells, ERK activity remained low and then increased rapidly when neutrophils spread over the endothelial cells. The high ERK activity was maintained during and after crawling, transmigration, and random migration between crypts. Fig. 1 E shows the activity change of ERK in a representative neutrophil, which incidentally migrated in the same XY plane, thereby allowing us to simultaneously trace the movement along with the activity change. To quantify these observations, neutrophils in each step were randomly selected in the CFP images and then analyzed for their ERK activity in the FRET/CFP ratio image. Clearly, the ERK activity was increased between the adhesion and crawling steps (Fig. 1 F).

\section{Requirement of ERK activity for neutrophils crawling over the endothelial cells and migrating in the interstitial tissue} The ERK activation during the adhesion of neutrophils prompted us to examine the effect of an MEK inhibitor, PD0325901, on the neutrophil recruitment cascade. PD0325901 was injected intravenously into Eisuke mice that had been subjected to LPS and AMLP injection. ERK activity in the 

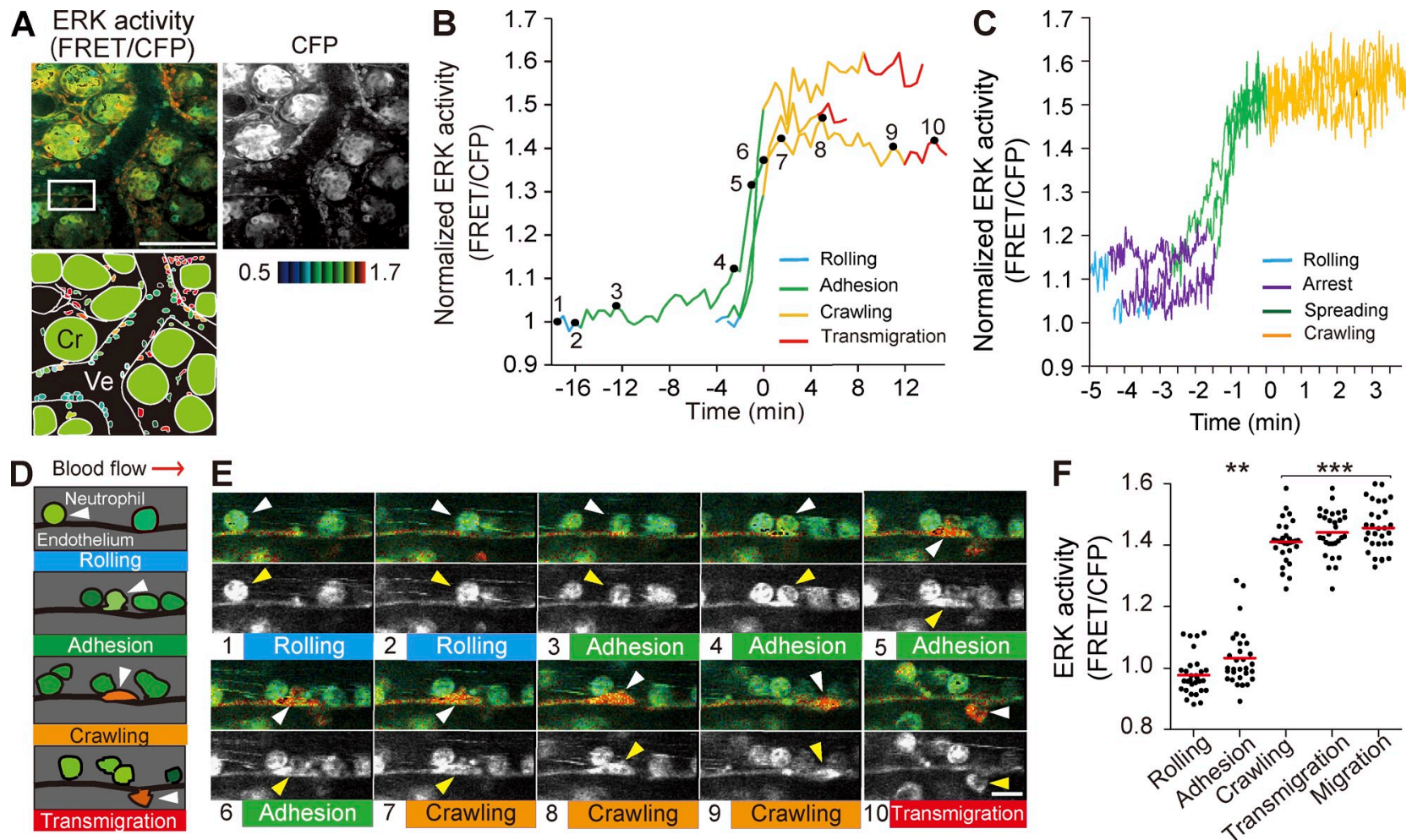

Figure 1. Activation of ERK in neutrophils during adhesion to endothelial cells of the inflamed small intestine. (A) In vivo imaging of the lamina propria of the intestinal mucosa from an Eisuke mouse, which was subjected to LPS and fMLP $2 \mathrm{~h}$ before imaging. The representative FRET/CFP ratio image is shown in intensity-modulated display mode with 32-intensity in 8-ratio and a CFP image in grayscale from Video 1, with a schematic view of this region. $\mathrm{Cr}$, crypt; Ve, venule. Gamma, 1.7. The image is representative of a mouse in five independent experiments. (B) ERK activities in three representative neutrophils from three independent experiments are plotted against time. The transition point from the adhesion to crawling steps was set as time 0 . The rolling, adhesion, crawling, and transmigration steps are indicated by different colors. (C) ERK activities from three representative neutrophils were recorded with a shorter interval than in $\mathrm{B}$, at one image every $1.5 \mathrm{~s}$, to discriminate arrest and spreading phases of the adhesion step. Neutrophils were defined as arrest when they stopped rolling for $>30 \mathrm{~s}$. The start of spreading was defined when neutrophils changed from a round to an amoeboid shape. (D) Schematic of the four steps of extravasation. Arrowheads indicate the same neutrophil at different time points. (E) Time-lapse FRET/CFP and CFP images of neutrophil extravasation. The boxed region in A was magnified and shown in a time series. The arrowheads indicate the same neutrophil traced in the video. Bars: (A) $100 \mu \mathrm{m}$; (E) $10 \mu \mathrm{m}$. (F) ERK activity of neutrophils during neutrophil recruitment to the inflamed tissue. 30 neutrophils in each step were randomly selected in the CFP images and examined for their ERK activity in the corresponding FRET/CFP ratio image. Results obtained from three mice are combined. Black dots and red bars indicate the ERK activity in each neutrophil and the mean values, respectively. Difference from the rolling cells was evaluated by the Student's $t$ test: ${ }^{*}, \mathrm{P}<0.01$; ***, $P<0.001$

interstitial neutrophils dropped within 20 min after PD0325901 administration to the level of the averaged ERK activity of neutrophils in the venules (Fig. 2, A and B; and Video 2). PD0325901 treatment did not significantly change the total number of neutrophils associated with the venules but decreased the number of neutrophils crawling over or transmigrating through the endothelial cells (Fig. 2 C). The proportion of neutrophils rolling over or adhering to the endothelial cells was increased accordingly. The effects on rolling and adhesion of neutrophils were further quantified as described previously (Fig. 2, D and E; Kubes et al., 2003). We found that PD0325901 profoundly increased the number of adherent neutrophils. These results agree with the finding that ERK activity increased before the initiation of crawling.

The effect of PD0325901 on each step of the recruitment cascade was analyzed in detail with time-lapse video (Video 2).
After binding to the endothelial cells, about half of neutrophils were detached within $15 \mathrm{~s}$ (Fig. 2 F). Before the addition of PD0325901, the detachment rate was slowed down after $30 \mathrm{~s}$, which corresponded to the transition to the crawling phase. In contrast, after the injection of PD0325901, the neutrophils on the endothelial cells decreased exponentially. The neutrophils in the presence of PD0325901 crawled over and transmigrated through the endothelial cells more slowly than did the neutrophils in the absence of PD0325901 (Fig. 2, $\mathrm{G}$ and $\mathrm{H}$ ). As is evident in Video 2, the neutrophils in the interstitial tissue also slowed down after PD0325901 treatment (Fig. 2 I). These data clearly showed that the high ERK activity of the neutrophils promotes crawling over and transmigrating through the endothelial cells and random migration in the interstitial tissues. 
A

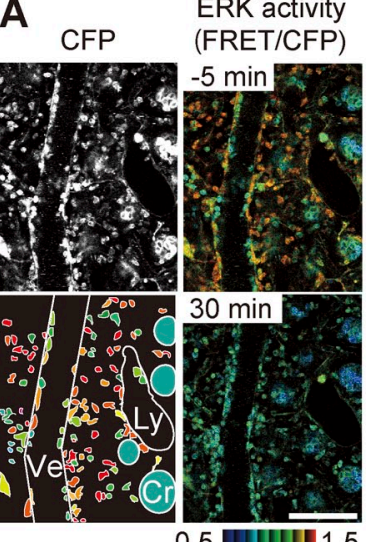

0.5 ㅂIIIIII 1.5

E
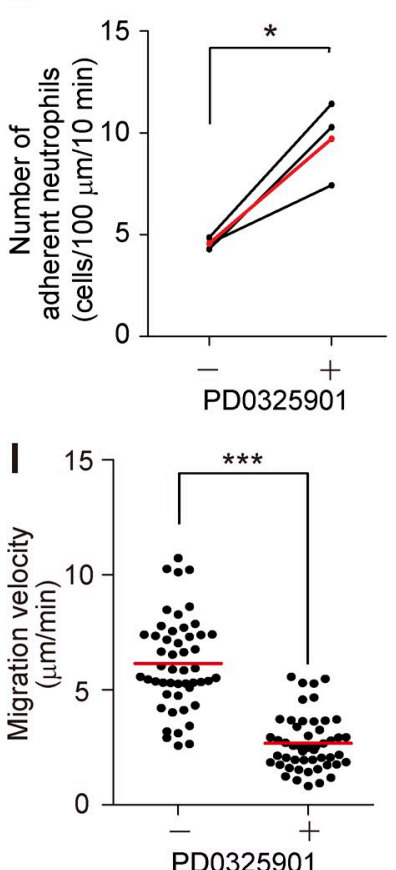

B 1.5 PD0325901

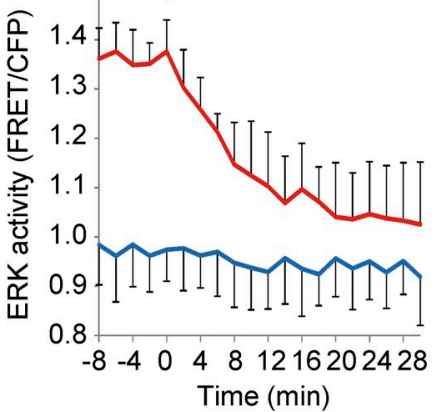

- Interstitial neutrophis

- Intravascular neutrophils

$\mathbf{F}$
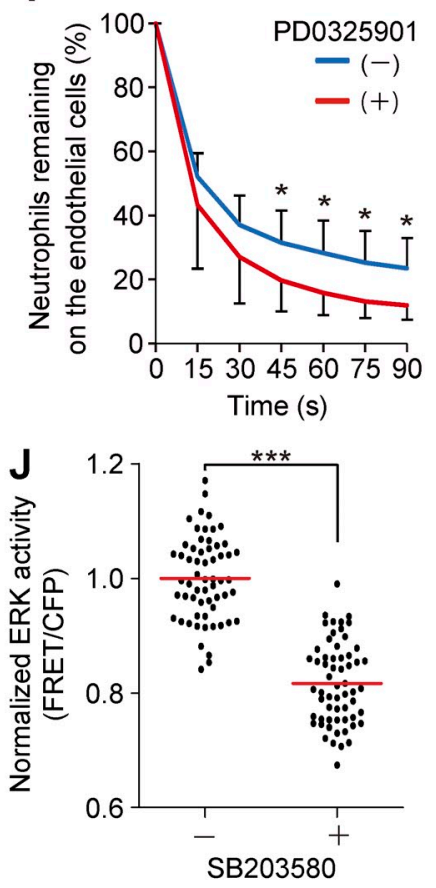

C
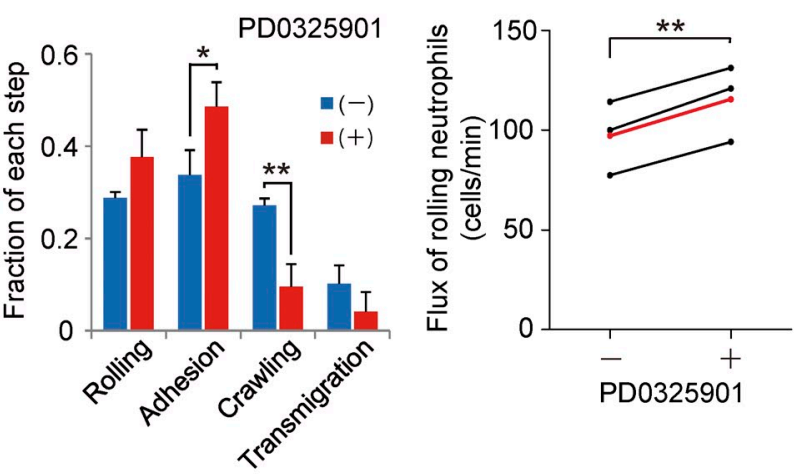

G
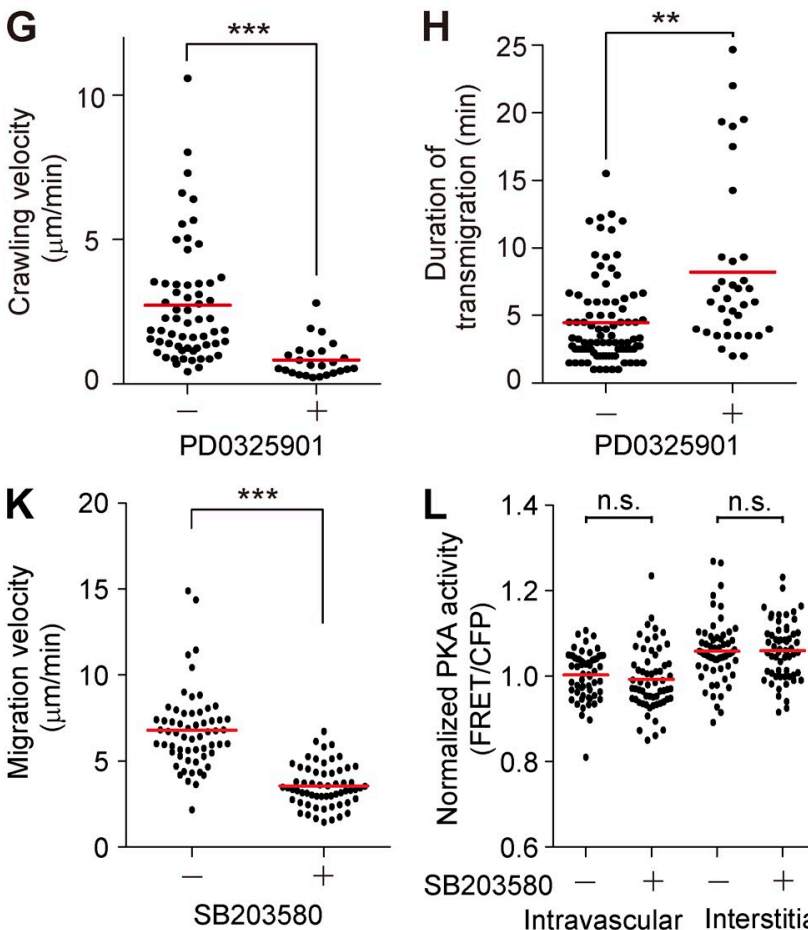

Figure 2. Requirement of ERK activity for the neutrophil recruitment. (A) FRET images of the lamina propria of the intestinal mucosa in Eisuke mice $2 \mathrm{~h}$ after LPS and fMLP treatment. At time $0,5 \mathrm{mg} / \mathrm{kg}$ of the PD0325901 MEK inhibitor was injected intravenously. A CFP image and two FRET/CFP images are cropped from Video 2, with a schematic view of this region. Cr, crypt; Ly, lymphatic vessel; Ve, venule. FRET/CFP images are prepared with the scale range shown at the bottom. Gamma, 1.7. The image is representative of a mouse in five independent experiments. Bar, $100 \mu \mathrm{m}$. (B) Time courses of the ERK activity of intravascular or interstitial neutrophils. In each of three mice, 10 neutrophils in and out of the venules were randomly selected in the CFP images and examined for their ERK activity in the corresponding FRET/CFP ratio image. The mean and one SD of the average of each mouse are shown. (C) Neutrophils on the endothelial cells were classified into the four steps before and after PD0325901 treatment. We scored 152 and 147,148 and 153, and 137 and 129 neutrophils before and after PD0325901 treatment in three mice. Error bars indicate the SD. ${ }^{*}, P<0.05{ }^{*}{ }^{*}, P<0.01$ (Student's $t$ test). (D and E) Eisuke mice pretreated with LPS and fMLP were inoculated with the MEK inhibitor PD0325901 and imaged every $1.5 \mathrm{~s}$. Rolling leukocyte flux (D) or number of adherent neutrophils (E) was quantified as described previously (Kubes et al., 2003). Three mice were analyzed independently, and the mean values are indicated in red. ${ }^{*}, P<0.05{ }^{*}{ }^{*}, P<0.01$ (paired Student's $t$ test). (F) The duration of neutrophil attachment to the endothelial cells was examined before and after PD0325901 treatment ( $n>150$ for each condition). Data were collected from four mice. Error bars indicate the SD. Asterisks indicate the result of the paired Student's $t$ test between each time point and time $0:{ }^{*}, \mathrm{P}<0.05$. $(\mathrm{G}$ and $\mathrm{H})$ Velocity of crawling over the endothelial cells $(G)$ and the duration of transmigration $(H)$ were measured for neutrophils that could be tracked during 30-min time-lapse imaging either before or after PD0325901 treatment. Data obtained from three mice were combined and plotted. Dots and bars indicate the crawling velocity (G) and duration of transmigration $(\mathrm{H})$ of each neutrophil and the mean values, respectively. ${ }^{* *}, \mathrm{P}<0.01 ;{ }^{* *}, \mathrm{P}<0.001$ (Mann-Whitney $U$ test). (I) Migration velocity of interstitial neutrophils was measured before and after PD0325901 treatment. 50 neutrophils from three mice were randomly selected in the CFP images and examined for migration velocity during 5-min time-lapse imaging. Dots and bars indicate the migration velocity of each neutrophil and the mean values, 

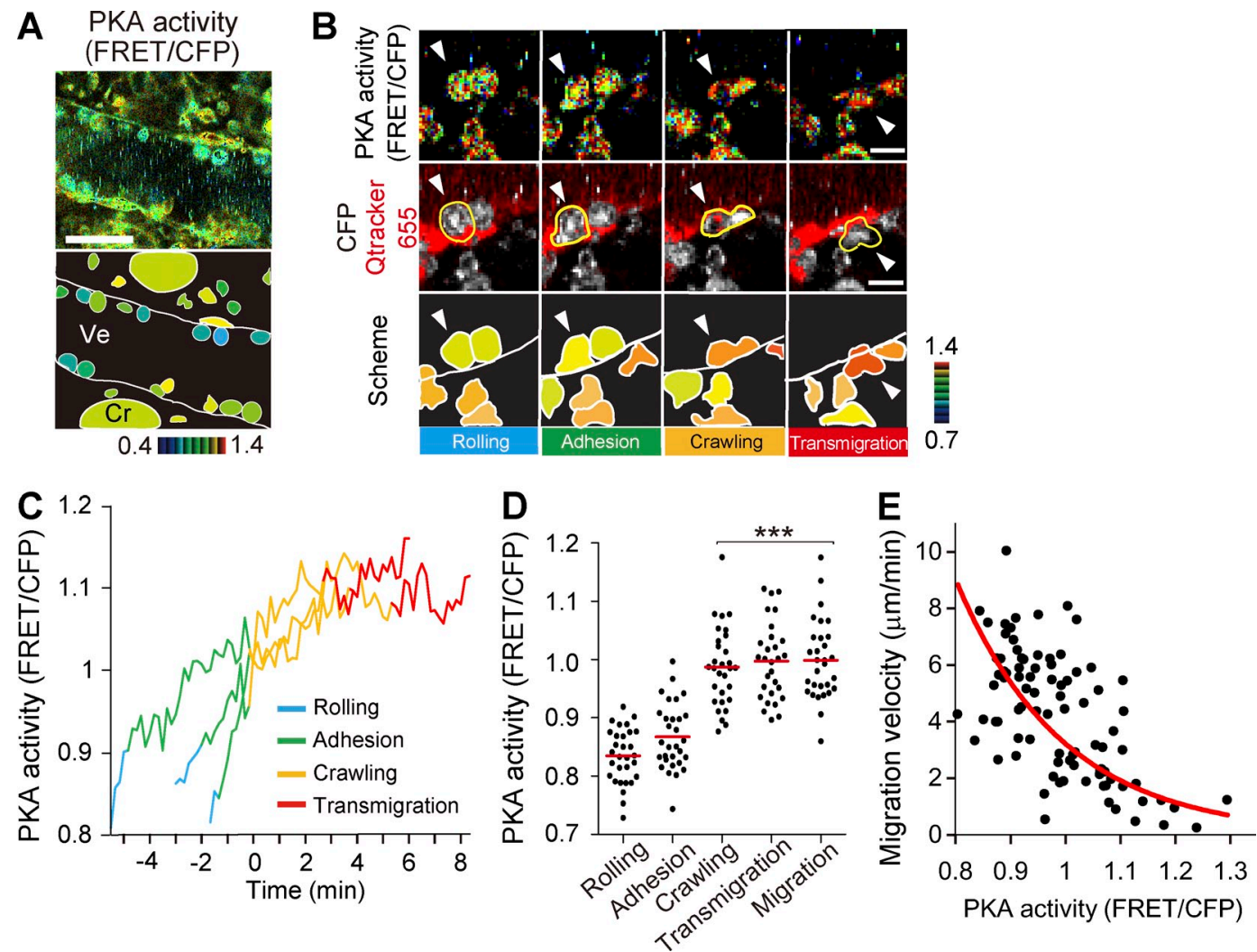

Figure 3. Activation of PKA during the neutrophil recruitment cascade. (A) In vivo imaging of the lamina propria of the intestinal mucosa of PKAchu mice. A representative FRET/CFP ratio image from Video 3 and a scheme are shown. $\mathrm{Cr}$, crypt; Ve, venule. Gamma, 1.14. The image is representative of a mouse in five independent experiments. (B) In vivo imaging of the lamina propria of the intestinal mucosa of a C57BL/6 mouse that was transplanted with bone marrow of PKAchu mice 7 wk before the experiment. Representative FRET/CFP ratio images and CFP/Qtracker 655 images from Video 4 and schemes during neutrophil extravasation are shown. Tracking of a neutrophil is shown by arrowheads. The same cells were also marked by yellow lines. Gamma, 1.44. The image is representative of a mouse in three independent experiments. Bars: (A) $30 \mu \mathrm{m}$; (B) $10 \mu \mathrm{m}$. (C) The PKA activity change was monitored in three representative neutrophils from three independently analyzed mice. The time of transition from the adhesion to crawling steps was set as zero. The rolling, adhesion, crawling, and transmigration steps are indicated by different colors. (D) PKA activity of neutrophils in the four steps of extravasation is plotted. 30 neutrophils in each step were randomly chosen in the CFP images and examined for their PKA activity in the corresponding FRET/CFP ratio image. To accumulate the incidence, three independent experiments were performed. Dots and bars indicate the PKA activity in each neutrophil and the mean values, respectively. ${ }^{* *}, P<0.001$ (Student's $t$ test). (E) 90 neutrophils migrating in the interstitial tissue were randomly chosen in the CFP images and examined for their PKA activity and migration velocity during 5 min of time-lapse imaging. Results obtained from three mice were combined. The red line is an approximate curve showing the inverse correlation between PKA activity and migration velocity.

Recently, it was reported that neutrophil migration in vitro is regulated negatively by ERK and positively by p38 MAPK (Liu et al., 2012). It has also been shown that the end target chemoattractants such as $\mathrm{MLP}$ and C5a require p38 MAPK for their action (Heit et al., 2002). We found that a p38 MAPK inhibitor, SB203580, inhibited ERK activity and migration of neutrophils without affecting PKA activity (Fig. 2, J-L), suggesting that p38 MAPK may control neutrophil migration in an ERK-dependent and PKA-independent manner.

\section{PKA activity in the neutrophils during} the neutrophil recruitment cascade and migration

Neutrophils at the inflammatory sites perceive signals from chemokines and prostaglandins, which transduce signals primarily via $G_{i^{-}}$and $G_{s}$-coupled receptors, respectively. To examine which of the $\mathrm{G}_{\mathrm{i}^{-}}$or $\mathrm{G}_{\mathrm{s}}$-mediated signaling cascades is dominant in the neutrophils during the neutrophil recruitment cascade, we examined PKA activity in the small intestines of PKAchu mice, which expressed a cytoplasmic FRET

respectively. ${ }^{* * *}, \mathrm{P}<0.001$ (Mann-Whitney $U$ test). (J-L) Inhibition of ERK activity by a p38 inhibitor, SB203580. Eisuke mice or PKAchu mice pretreated with LPS and fMLP were inoculated with $15 \mathrm{mg} / \mathrm{kg}$ SB203580. 60 neutrophils from three mice were randomly selected in the CFP images and examined for ERK activity (J), migration velocity during 5-min time-lapse imaging (K), and PKA activity (L). Dots and bars indicate the ERK activity (J), migration velocity $(K)$, and PKA activity $(L)$ of each neutrophil and the mean values, respectively. Data were acquired 10 min before and 20 min after SB203580 injection. ${ }^{* *}, \mathrm{P}<0.001$; n.s., not significant ( $\mathrm{J}$ and L, Student's $t$ test; $\mathrm{K}$, Mann-Whitney $U$ test). 

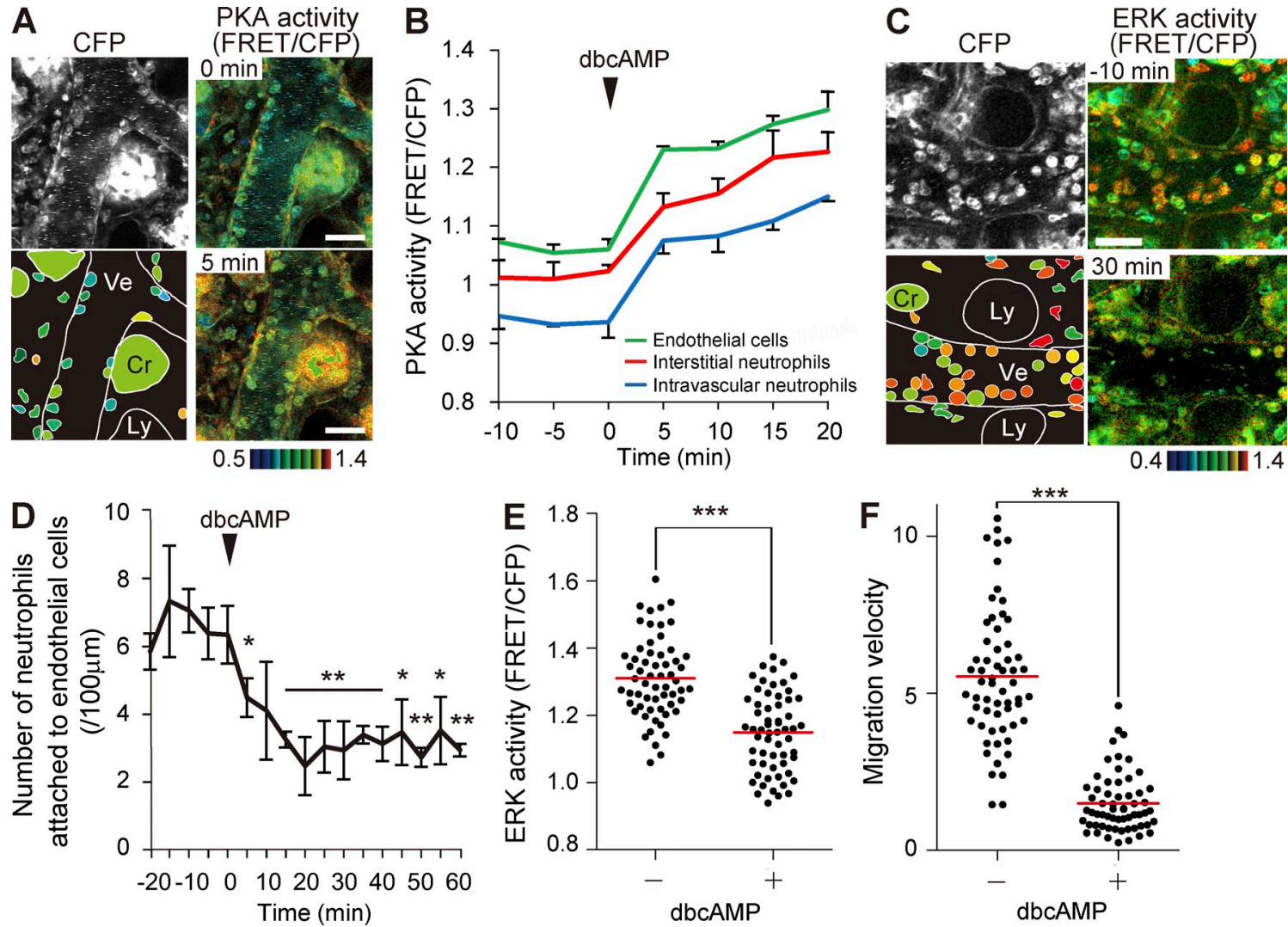

Figure 4. PKA inhibition of ERK activity, recruitment to endothelial cells, and migration of neutrophils. (A) FRET images of the lamina propria of the intestinal mucosa in PKAchu mice pretreated with LPS and fMLP. A cAMP analogue, dbcAMP, was injected intravenously at $0.3 \mathrm{~g} / \mathrm{kg}$ at time 0 . The bottom left panel shows the schematic view of this region. Cr, crypt; Ly, lymphatic vessel; Ve, venule. Gamma, 1.7. The image is representative of a mouse in three independent experiments. (B) Time courses of the PKA activity of intravascular and interstitial neutrophils and endothelial cells. In each of three mice, 10 neutrophils in and out of the venules and 10 endothelial cells were randomly selected in the CFP images and examined for PKA activity. Mean data of three mice are shown with one SD. (C) FRET images of the lamina propria of the intestinal mucosa in Eisuke mice pretreated with LPS and fMLP. A cAMP analogue, dbcAMP, was injected intravenously $(0.3 \mathrm{~g} / \mathrm{kg})$ at time 0 . Images are cropped from Video 5 . Gamma, 1.2. The image is representative of a mouse in three independent experiments. (A and C) Bars, $30 \mu \mathrm{m}$. (D) Inhibition of the neutrophil attachment to the endothelial cells by dbcAMP treatment. The number of neutrophils on the endothelial cells was counted in three mice, and the mean value and one SD of the average of each mouse are plotted against time. Asterisks indicate the result of the paired Student's $t$ test between each time point and time $0:{ }^{*}, P<0.05 ;{ }^{* *}, P<0.01$. (E and F) Correlation of ERK activity and migration velocity of interstitial neutrophils before and after dbcAMP treatment. 60 neutrophils that were imaged in three mice were randomly selected in the CFP images before and after dbcAMP treatment and examined for their ERK activity (E) and migration velocity (F) during 5 min of time-lapse imaging. Red bars indicate the mean values. ${ }^{* *}, \mathrm{P}<0.001$ ( $\mathrm{E}$, Student's $t$ test; $\mathrm{F}$, Mann-Whitney $U$ test).

biosensor for PKA, AKAR3EV (Fig. 3 A and Video 3). Unlike the neutrophils of Eisuke mice, the expression of the FRET biosensor for PKA was relatively low in PKAchu mice.Therefore, in some experiments, C57BL/6 mice were transplanted with bone marrow of PKAchu mice 7 wk before analysis. In this way, we could eliminate the fluorescence from endothelial cells and mesenchymal cells and confirm the observations obtained with PKAchu mice (Fig. 3 B andVideo 4). The PKA activity of neutrophils rolling on the endothelial cells was lower than that of the neutrophils that were adhering to, crawling over, or transmigrating through the endothelial cells (Fig. 3, C and D). In contrast to ERK activity, which showed a rapid increase before entering into the crawling step, PKA activity increased gradually during the neutrophil recruitment cascade (Fig. 3 C). After emigration to the interstitial tissue, the PKA activity in neutrophils varied cell by cell. Notably, the PKA activity was inversely correlated with the migration velocity of each neutrophil in the interstitial tissue (Fig. 3 E). These data suggested that PKA activity negatively regulated migration in the interstitial tissue.

\section{PKA inhibition of ERK activity, recruitment} to endothelial cells, and migration of neutrophils

The role of PKA in the neutrophil recruitment cascade was further examined by intravenous injection of a cAMP analogue, dibutyryl cAMP (dbcAMP). PKA was rapidly activated in almost all cell types in the lamina propria of the small intestine, including neutrophils, endothelial cells, and epithelial cells (Fig. 4, A and B). In clear contrast, ERK activity was decreased in neutrophils with a slightly slower time course (Fig. $4 \mathrm{C}$ and 
A

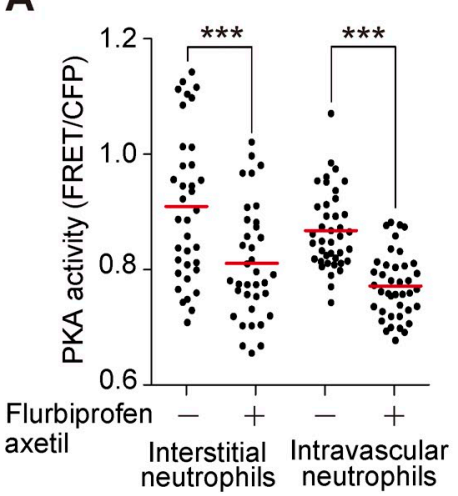

B

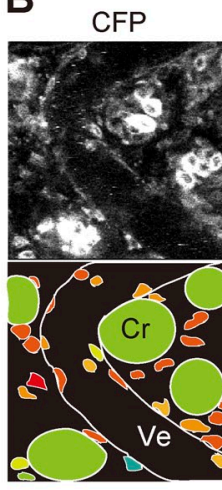

ERK activity (FRET/CFP) $-10 \min 30$

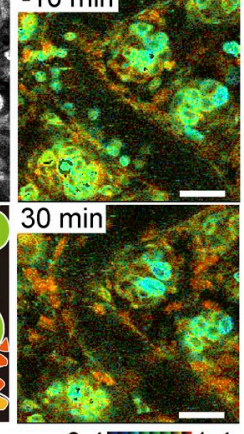

C Flurbiprofen axetil

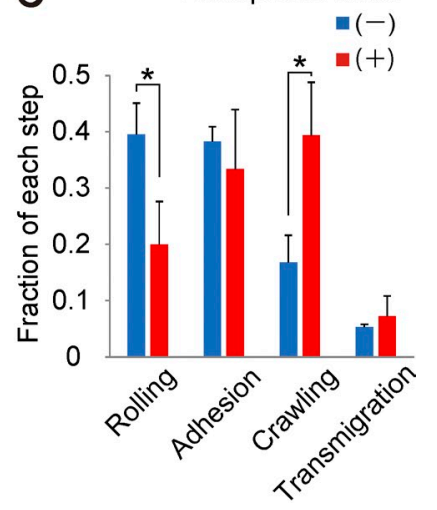

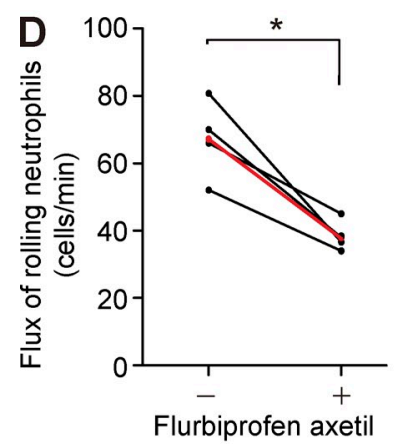

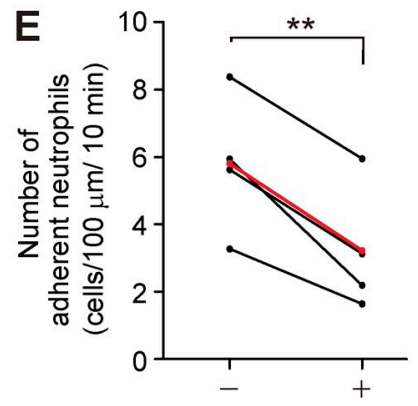

Flurbiprofen axetil

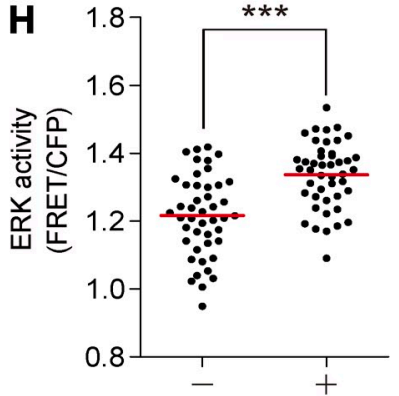

Flurbiprofen axetil

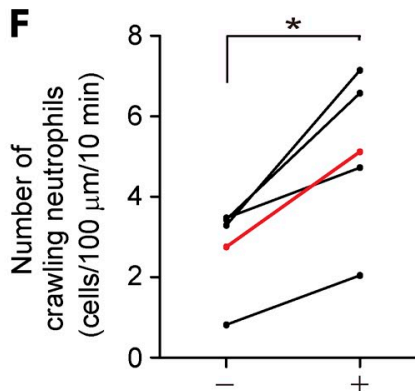

Flurbiprofen axetil

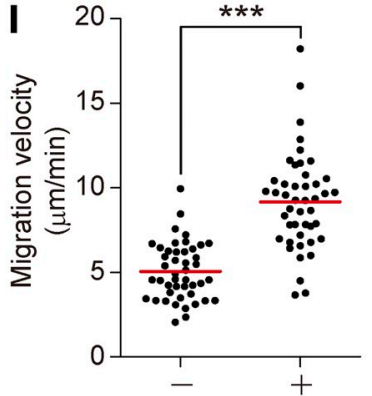

Flurbiprofen axetil

Figure 5. Accelerated migration and activation of ERK in neutrophils treated with NSAID. (A) PKA activity of intravascular and interstitial neutrophils before and after $7.5 \mathrm{mg} / \mathrm{kg}$ flurbiprofen axetil injection. 40 neutrophils in and out of venules were randomly selected in the CFP images of three mice and examined for the PKA activity in the corresponding FRET/CFP ratio images. Red bars indicate mean values. ${ }^{* *}, \mathrm{P}<0.001$ (Student's $t$ test). (B) FRET images of the lamina propria of the intestinal mucosa in Eisuke mice pretreated with LPS and fMLP and injected with $7.5 \mathrm{mg} / \mathrm{kg}$ flurbiprofen axetil at time 0 . Images are cropped from Video 6. A CFP image and a scheme are also shown. $\mathrm{Cr}$, crypt; Ve, venule. Gamma, 1.3. The image is a representative view field of a mouse in three independent experiments. Bars, $30 \mu \mathrm{m}$. (C) Increase in the crawling step and decrease in the rolling step in the neutrophil recruitment cascade by flurbiprofen axetil treatment. The neutrophils on the endothelial cells were categorized into the four steps of the neutrophil recruitment cascade. The fraction of each step was calculated after summing up three time points each for before and after flurbiprofen axetil treatment. The experiment was repeated three times, and 537 and 503 neutrophils in total were scored before and after flurbiprofen axetil injection, respectively. Error bars indicate the SD. ${ }^{*}, \mathrm{P}<0.05$ (Student's $t$ test). (D-G) Acceleration of neutrophil crawling and transmigration by flurbiprofen axetil injection. Eisuke mice pretreated with LPS and fMLP were time-lapse imaged before and after intravenous administration of flurbiprofen axetil. The images were acquired every $1.5 \mathrm{~s}$ for the detailed analysis. The flux of rolling neutrophils (D) and the number of adherent (E), crawling (F), or transmigrating neutrophils (G) were quantified with time-lapse essentially as described previously (Kubes et al., 2003). A neutrophil was defined as adherent to endothelial cells if it remained stationary for $>30 \mathrm{~s}$. The numbers of adherent, crawling, or transmigrating neutrophils were averaged per 10-min observation period. Four mice were analyzed independently, and mean values are indicated in red. ${ }^{*}, P<0.05 ;{ }^{* *}, P<0.01$ (paired Student's $t$ test). (H and I) The effect of flurbiprofen axetil on the ERK activity and migration velocity of interstitial neutrophils. 60 neutrophils were randomly selected in the CFP images at -20 and $20 \mathrm{~min}$, and the migration velocity of each neutrophil was measured. Three independent experiments were performed to collect the data. Dots indicate the ERK activity $(\mathrm{H})$ and migration velocity $(\mathrm{I})$ in each neutrophil, and red bars indicate the mean values. ***, $\mathrm{P}<0.001$ (Student's $t$ test and Mann-Whitney $U$ test are used for the ERK activity [H] and migration velocity [I], respectively). 
A

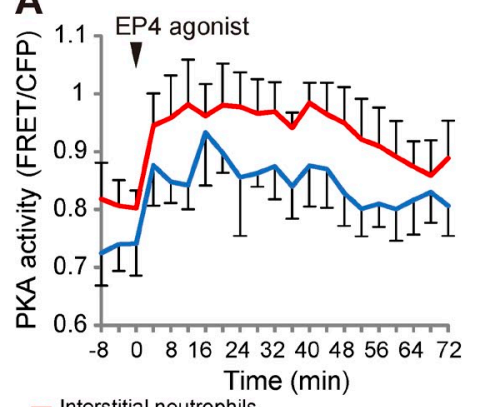

- Interstitial neutrophils
- Intravascular neutrophils

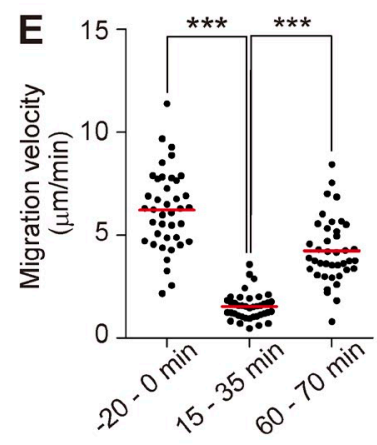

B

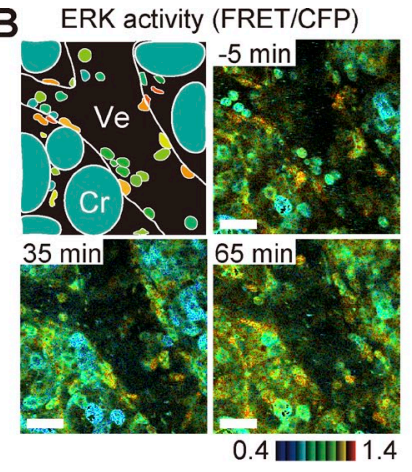

F Flurbiprofen

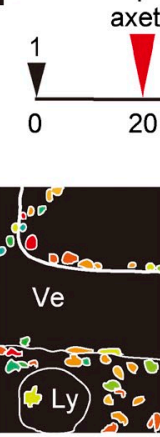

EP4 agonist

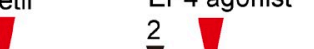

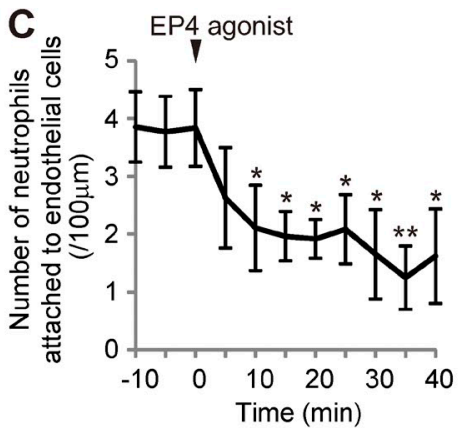

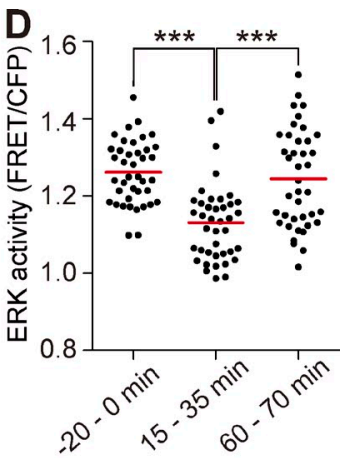

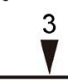
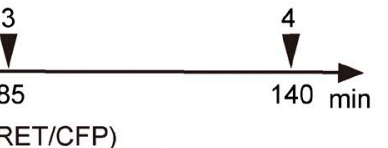

ERK activity (FRET/CFP)
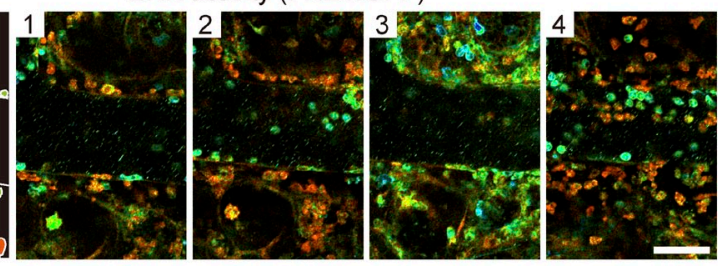

0.4 ㄸIIIII 1.5
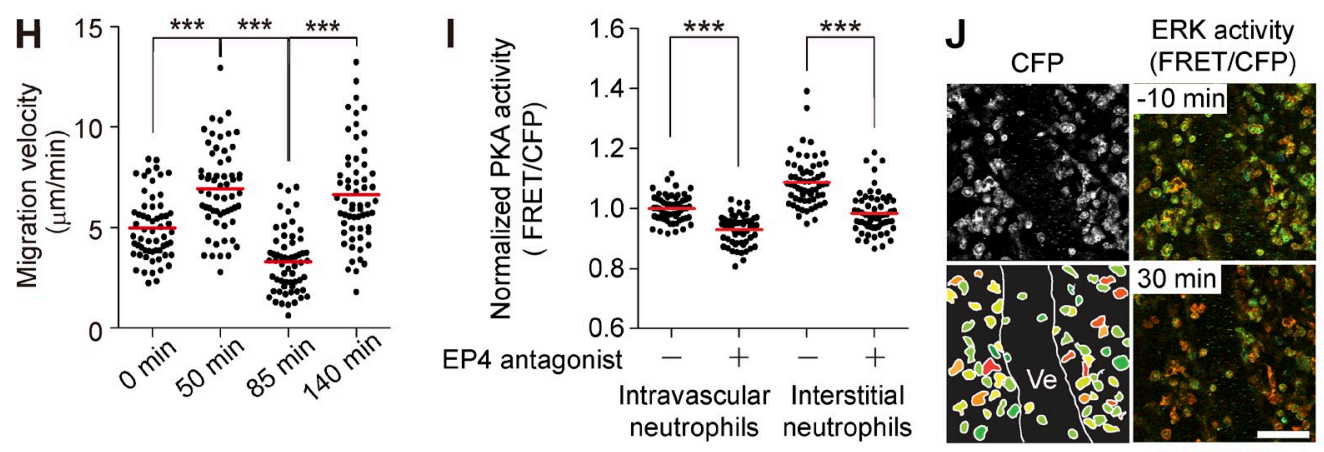

0.4 manกIII 1.4

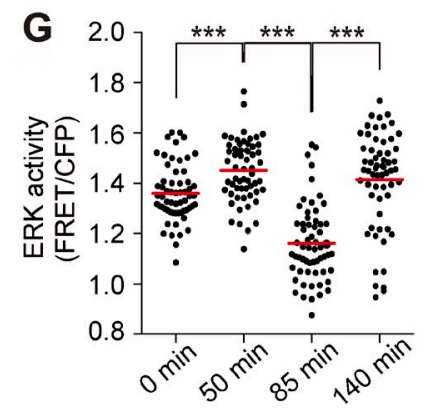

$\mathbf{L}$

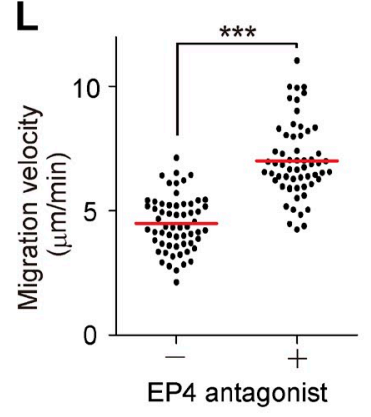

M

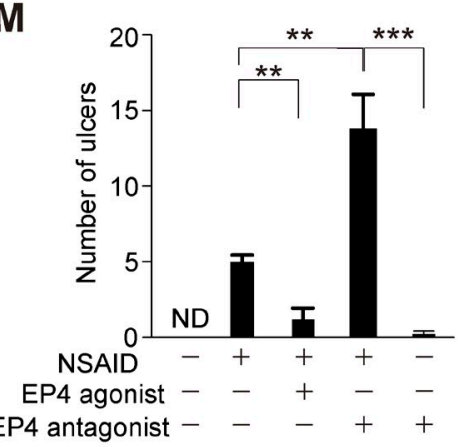

$\mathbf{N}$

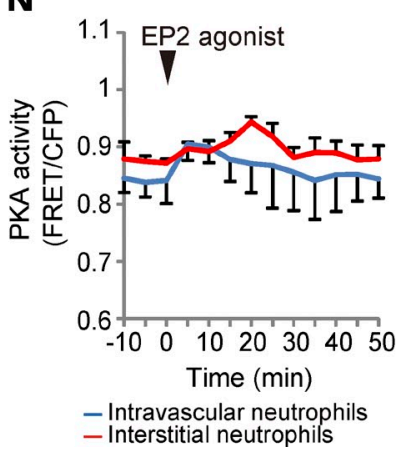

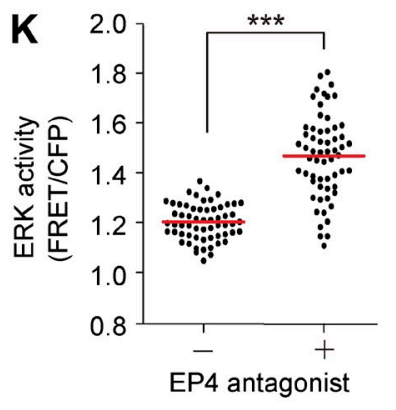

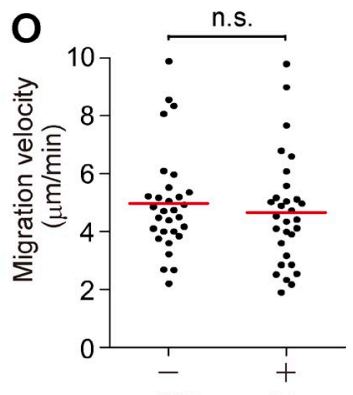

EP2 agonist

Figure 6. Inhibition of the recruitment to endothelial cells and the migration of neutrophils by an EP4 agonist. (A) Time courses of the PKA activity of intravascular and interstitial neutrophils are plotted against time. An EP4 agonist, ONO-AE1-329, was injected intravenously (0.25 mg/kg) at time 0.10 neutrophils in each group were randomly selected in the CFP images and examined for PKA activity in the corresponding FRET/CFP ratio image. Three mice were analyzed independently, and the mean and one SD are indicated. (B) In vivo imaging of the lamina propria of the intestinal mucosa in Eisuke mice and a schematic view of this region. Images are cropped from Video $7.0 .25 \mathrm{mg} / \mathrm{kg}$ ONO-AE1-329 was injected intravenously at time 0. Cr, crypt; Ly, lymphatic vessel; Ve, venule. Gamma, 1.3. The image is a representative view field of a mouse in three independent experiments. 
Video 5). Concomitant with the decrease in ERK activity, the number of neutrophils attached to the endothelial cells was also significantly decreased (Fig. 4 D). In the interstitial tissues, dbcAMP treatment also decreased the ERK activity and migration velocity of neutrophils (Fig. 4, E and F), strongly suggesting that PKA regulates neutrophils via negative regulation of ERK.

\section{Increase in ERK activity and migration velocity of interstitial neutrophils by nonsteroidal antiinflammatory drug (NSAID) administration}

We next examined the effect of an NSAID, flurbiprofen axetil, on the activities of ERK and PKA in the inflamed intestine. NSAIDs generally inhibit production of $\mathrm{PGE}_{2}$ and thereby suppress $G_{\mathrm{s}}$-coupled receptors such as EP2 and EP4. In agreement with a previous study showing the expression of EP2 and EP4 in neutrophils (Yamane et al., 2000), PKA activity was decreased in both intravascular and interstitial neutrophils within $10 \mathrm{~min}$ after intravenous flurbiprofen axetil injection (Fig. 5 A). In contrast, flurbiprofen axetil injection caused a gradual increase in the ERK activity of neutrophils (Fig. 5 B and Video 6). In the venules, the major fraction of neutrophils was shifted from the rolling step to the crawling step by flurbiprofen axetil (Fig. 5 C). Detailed analysis of neutrophils on the endothelial cells revealed that flurbiprofen axetil enhanced progression from the adhesion step to the crawling step (Fig. 5, D-G). Meanwhile, in the interstitial tissue, both the ERK activity and migration velocity of interstitial neutrophils were significantly increased within
20 min after flurbiprofen axetil injection (Fig. 5, H and I). We obtained a similar result with another NSAID, indomethacin, suggesting that the observed effects arose mostly through the inhibition of COXs (not depicted).

\section{Inhibition of neutrophil migration by EP4 activation}

The accelerated migration and increased ERK activation of neutrophils in the NSAID-treated intestine led us to examine the role of $\mathrm{PGE}_{2}$ on neutrophil migration. We first intravenously administered an EP4 agonist, ONO-AE1-329, to examine the role of EP4 on neutrophils. The EP4 agonist ONO-AE1-329 strongly and rapidly increased PKA activity and decreased ERK activity in intravascular and interstitial neutrophils and in endothelial cells (Fig. 6, A and B; and Video 7). Intravenous injection of ONO-AE1-329 gradually reduced the number of neutrophils on the endothelial cells, in agreement with the role of ERK and PKA in the recruitment of neutrophils (Fig. 6 C). Both the ERK activity and migration velocity of interstitial neutrophils were gradually restored within $60 \mathrm{~min}$ after ONO-AE1-329 treatment, when PKA activity also returned to almost the basal level (Fig. 6, A, D, and E). The effect of EP4 activation was more clearly demonstrated when flurbiprofen axetil was injected before ONO-AE1-329 administration (Fig. 6 F). ONO-AE1-329 markedly inhibited ERK activity and migration of interstitial neutrophils (Fig. 6, F-H; and Video 8). The effect was lost within $80 \mathrm{~min}$, probably as a result of inactivation in the lung. We next confirmed the role played by EP4 with an EP4 antagonist, ONO-AE3-208. As expected, intravenous injection of ONO-AE3-208 suppressed

(C) Inhibition of the entry of neutrophils into the neutrophil recruitment cascade by ONO-AE1-329 treatment. The numbers of neutrophils on the endothelial cells in three mice were counted, and the mean values with one SD are plotted against time. Bars indicate the SD. Asterisks indicate the result of the paired Student's $t$ test between each time point and time $0:{ }^{*}, P<0.05 ;{ }^{* *}, P<0.01$. (D and E) ERK activity and migration velocity of neutrophils migrating in the connective tissue at the indicated time points. 20 neutrophils in each of two mice were analyzed. Black dots and red bars indicate the ERK activity (D) and migration velocity (E) in each neutrophil and the mean values, respectively. ${ }^{* *}, P<0.001$ ( $D$, Student's $t$ test; $E$, Mann-Whitney $U$ test). (F) Cancellation of the effect of flurbiprofen axetil by ONO-AE1-329. FRET images of the lamina propria of the intestinal mucosa in Eisuke mice pretreated with LPS and fMLP. $7.5 \mathrm{mg} / \mathrm{kg}$ flurbiprofen axetil and $0.25 \mathrm{mg} / \mathrm{kg}$ ONO-AE1-329 were administered as indicated. Gamma, 1.14 . Images are cropped from Video 8. The image is a representative view field of a mouse in three independent experiments. ( $G$ and $H$ ) ERK activities and migration velocities of neutrophils in the interstitial tissue at the indicated time points. 60 neutrophils from three mice were analyzed. Black dots and red bars indicate the ERK activity $(\mathrm{G})$ and migration velocity $(\mathrm{H})$ in each neutrophil and the mean values, respectively. ${ }^{* *}, \mathrm{P}<0.001$ (Student's $t$ test). (I) PKA activity of intravascular and interstitial neutrophils $10 \mathrm{~min}$ before and $30 \mathrm{~min}$ after $5 \mathrm{mg} / \mathrm{kg} \mathrm{EP4} \mathrm{antagonist,} \mathrm{ONO-AE3-208,} \mathrm{injection.} 60$ neutrophils in and out of venules were randomly selected in the CFP images of three mice and examined for the PKA activity in the corresponding FRET/CFP ratio images. Red bars indicate mean values. Note that FRET values were normalized to the intravascular neutrophils in the absence of 0N0-AE3-208. ${ }^{* * *}, \mathrm{P}<0.001$ (Student's $t$ test). (J-L) Increase in ERK activity and migration velocity of neutrophils by ONO-AE3-208. (J) FRET images of the lamina propria of the intestinal mucosa in Eisuke mice pretreated with LPS and fMLP and injected with $5 \mathrm{mg} / \mathrm{kg}$ ONO-AE3-208 at time 0 . A CFP image and a scheme are also shown. Image is a representative view field of a mouse in three independent experiments. $(B, F$, and $K) B a r s, 50 \mu m$. (K and $L)$ The effect of ONO-AE3-208 on the ERK activity and migration velocity of interstitial neutrophils. 60 neutrophils were randomly selected in the CFP images at -10 and $30 \mathrm{~min}$, and ERK activity $(\mathrm{K})$ and the migration velocity $(\mathrm{L})$ of each neutrophil were measured. Three mice were used to collect the data. Dots indicate the ERK activity $(K)$ and migration velocity $(L)$ in each neutrophil, and red bars indicate the mean values. ${ }^{* * *}, P<0.001$ (Mann-Whitney $U$ test). (M) $\mathrm{C} 57 \mathrm{BL} / 6$ mice treated as indicated were sacrificed $24 \mathrm{~h}$ later to count the number of intestinal ulcers. Five mice were analyzed in each group. Error bars indicate the SD. ${ }^{* *}, P<0.01 ;{ }^{* * *}, P<0.001$ (Mann-Whitney $U$ test). ( $N$ and 0$)$ Effect of an EP2 agonist, ON0-AE1-259-01, on PKA activity of intravascular and interstitial neutrophils and migration velocity of interstitial neutrophils. (N) Time courses of PKA activity of intravascular and interstitial neutrophils are plotted against time. An EP2 agonist, $0.5 \mathrm{mg} / \mathrm{kg}$ ONO-AE1-259-01, was injected intravenously at time 0.30 neutrophils were randomly selected in the CFP images and examined for PKA activity in the corresponding FRET/CFP ratio image. Three independent experiments were performed, and the mean values with one SD are shown. (0) The effect of ONO-AE1-259-01 on the migration velocity of interstitial neutrophils. 30 neutrophils from three mice were randomly selected in the CFP images at -20 and $20 \mathrm{~min}$, and the migration velocity of each neutrophil was measured. Dots and bars indicate migration velocities in each neutrophil and mean values, respectively. n.s., not significant (Student's $t$ test). 
PKA, activated ERK, and promoted migration of neutrophils (Fig. 6, I-L). These results strongly suggest that $\mathrm{PGE}_{2}$-engaged EP4 inhibits ERK and thereby serves as an antiinflammatory signal. Because NSAIDs such as flurbiprofen axetil are known to cause ulcers in intestines, we examined the effect of ONO-AE1-329 and ONO-AE3-208 on the NSAID enteritis (Fig. $6 \mathrm{M}$ ). ONO-AE1-329 markedly decreased the number of flurbiprofen axetil-induced ulcers, whereas ONOAE3-208 increased the number of ulcers, although ONOAE3-208 alone could not cause ulcers. Thus, NSAID-mediated suppression of the EP4 signaling in the neutrophils seems to contribute at least partially to the development of NSAID enteritis. Of note, the EP2 agonist ONO-AE1-259-01 activated PKA only slightly and did not affect the migration velocity of the interstitial neutrophils (Fig. 6, $\mathrm{N}$ and $\mathrm{O}$ ).

\section{Inhibition of ERK and adhesion of neutrophils by an $\mathrm{LTB}_{4}$ receptor antagonist}

Finally, we examined the role played by $\mathrm{LTB}_{4}$, one of the major chemoattractants in acute inflammation, on ERK activity and the neutrophil recruitment cascade. Neutrophils express the high-affinity receptor for $\mathrm{LTB}_{4}$, BLT1, which is coupled with the heterotrimeric $\mathrm{G}_{\mathrm{i}}$ or $\mathrm{G}_{\mathrm{q}}$ protein (Yokomizo et al., 1997). We could not observe any effect of either $\mathrm{LTB}_{4}$ or an $\mathrm{LTB}_{4}$ receptor antagonist, LY293111, on the PKA activity in neutrophils (not depicted), suggesting that the effect of BLT1-mediated PKA inhibition is weak in neutrophils. In contrast, upon intravenous injection of $\mathrm{LTB}_{4}$, we observed a surge in ERK activity followed by rapid suppression (Fig. 7, A and B). The ERK activity correlated positively with the migration velocity of the interstitial neutrophils (Fig. 7 C). Next, $\mathrm{LTB}_{4}$ signaling was abrogated by an $\mathrm{LTB}_{4}$ receptor antagonist, LY293111. Upon intravenous injection of LY293111, ERK activity was gradually decreased in the interstitial neutrophils (Fig. 7, D and E). LY293111 significantly decreased the fraction of neutrophils in the crawling step and thereafter (Fig. 7 F) and inhibited the migration velocity of the interstitial neutrophils (Fig. 7 G). These results suggest that LTB $_{4}$ drives neutrophil emigration from the venules and migration in the interstitial tissues and that $\mathrm{LTB}_{4}$ was the canonical ERK activator of the neutrophils in the LPS-induced enteritis model. In conclusion, in vivo FRET imaging has revealed that $\mathrm{G}_{\mathrm{i}}$-coupled $\mathrm{LTB}_{4}$ activates ERK and transduces a "go" signal to neutrophils, whereas $\mathrm{G}_{\mathrm{s}}$-coupled EP4 activates PKA and thereby puts the brakes on neutrophil migration by suppressing ERK activity (Fig. $7 \mathrm{H}$ ).

\section{DISCUSSION}

ERK plays a critical role in cytokine-mediated neutrophil adhesion and aggregation in vitro (Pillinger et al., 1996; Capodici et al., 1998). However, the precise roles of ERK in the neutrophil recruitment to the inflammatory sites have not been shown in vivo as a result of technical difficulties. Here, we overcame this limitation by using Eisuke mice, which expressed a cytoplasmic FRET biosensor for ERK. The results showed that ERK in the vascular neutrophils was increased rapidly during adhesion to the endothelial cells (Video 1). This observation agreed with the previous in vitro finding that chemokines activate ERK and induce homotypic adhesions of neutrophils (Pillinger et al., 1996). Importantly, however, ERK activity also promoted the subsequent steps of chemotaxis to the inflammatory sites. We observed that an MEK inhibitor, PD0325901, suppressed not only crawling over and transmigration through the endothelial cells, but also migration in the interstitial tissues (Fig. 2). Thus, ERK plays a critical role in transducing "go" signals to the neutrophils, not only during the emigration from venules, but also during the migration in the interstitial tissues.

Recently Liu et al. (2012) showed that neutrophil migration in vitro is regulated negatively by ERK and positively by 38 MAPK and proposed that the dynamic balance between ERK and p38 controlled the neutrophil "stop" and "go" activity. This proposal contradicts our present findings and previous studies in which ERK activity was required for neutrophil migration in vitro (Pillinger et al., 1996; Capodici et al., 1998). In fact, the p38 inhibitor SB203580 that was used in the previous study inhibited ERK in neutrophils (Fig. 2, $\mathrm{J}-\mathrm{L}$ ). Thus, p38 may promote neutrophil migration in an ERK-dependent manner in vivo. The high concentrations of fMLP used by Liu et al. (2012) might have caused the discrepancy in the in vitro observations. Alternatively, several cytokines and/or growth factors that act on single neutrophils in vivo could bring about different outcomes. In any event, our observation with FRET mice highlighted the importance of in vivo imaging.

NSAIDs can injure the gastrointestinal tract, including the small intestine or colon (Bjarnason et al., 1993; Felder et al., 2000; Wallace, 2012). Neutrophils accumulate in the NSAIDinjured small intestine as soon as $6 \mathrm{~h}$ after NSAID administration (Stadnyk et al., 2002). Such mucosal injury induced by NSAIDs has been reported to be neutrophil dependent (Wallace et al., 1990). However, how neutrophils are activated by NSAID administration remains elusive. In the present study, we demonstrated that NSAID treatment activated the crawling step of the neutrophil recruitment process and migration of interstitial neutrophils along with the increase of ERK activity (Fig. 5). This observation strongly suggests that the NSAID-induced suppression of $\mathrm{PGE}_{2}$-mediated PKA activation results in ERK activation, which causes dissemination of active neutrophils from the inflammatory sites. Notably, an in vitro study showed that salicylates, but not indomethacin, inhibited ERK and homotypic aggregation of neutrophils without inhibiting COX (Pillinger et al., 1998). Therefore, monitoring of PKA activity is essential for assessment of the effects of NSAIDs in vivo. For this purpose, the PKAchu mice, which express a biosensor for PKA, will serve as an ideal model animal.

Another in vivo finding that contradicts the previous in vitro studies concerns the role of EP4. Although EP4 is associated with $G_{s}$ and may stimulate PKA, engagement of EP4 has been shown to activate ERK in many cell types. For example, EP4 but not EP2 signaling leads to phosphorylation of 


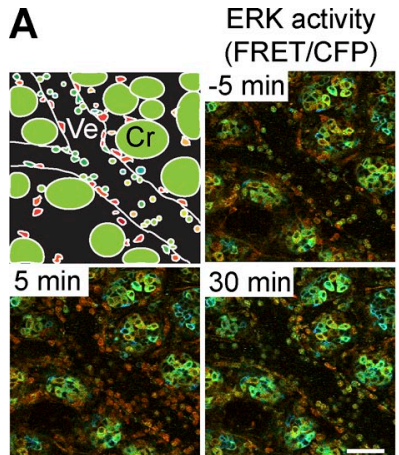

0.4 ㅂIIIII 1.4

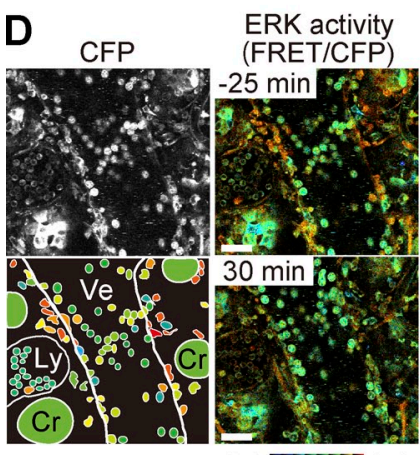

0.4 ㅂIIIIII 1.4
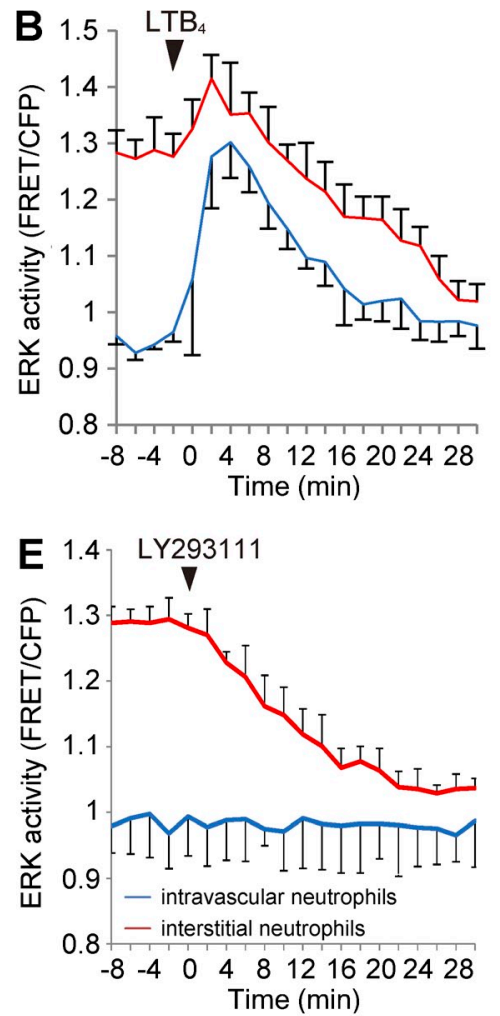

$\mathrm{H}$

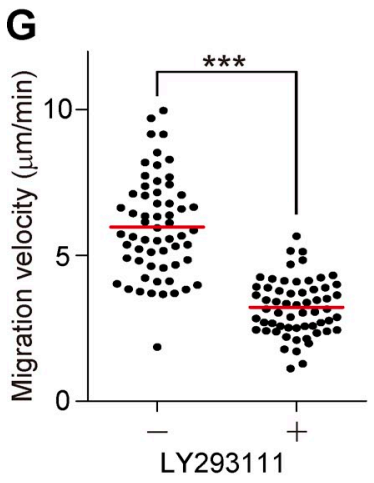

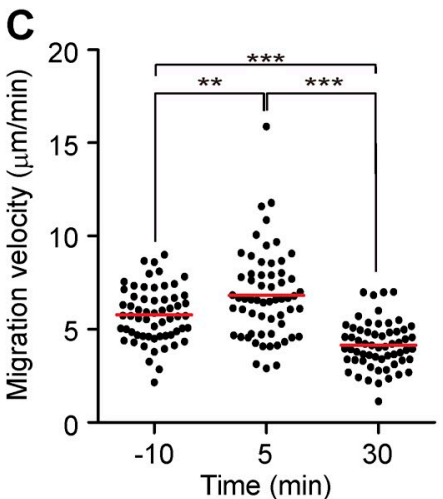

$\mathbf{F}$

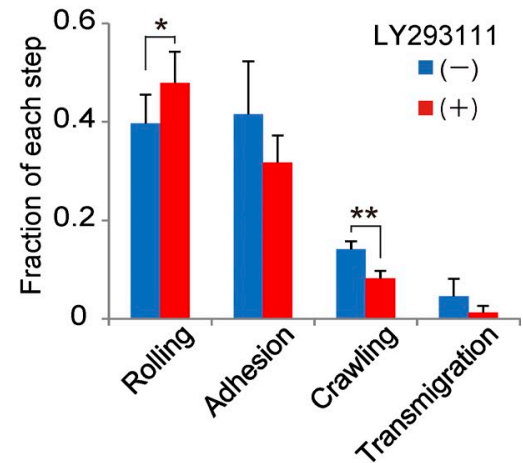

Figure 7. Effect of $\mathrm{LTB}_{4}$ and an $\mathrm{LTB}_{4}$ receptor antagonist on the ERK activity and the neutrophil recruitment. (A) FRET and CFP images of the lamina propria of the intestinal mucosa in Eisuke mice. The top left panel shows a schematic view of this image. At time 0 during time-lapse imaging, $\mathrm{LTB}_{4}$ was injected intravenously at $7.5 \mu \mathrm{g} / \mathrm{kg}$. Gamma, 1.14. Image is a representative view field of a mouse in three independent experiments. (B) Time courses of the ERK activity of intravascular and interstitial neutrophils plotted against time. 10 neutrophils in each of three mice were randomly selected in the CFP images and examined for ERK activity in the corresponding FRET/CFP ratio image. Error bars indicate the one SD. (C) Migration velocity of interstitial neutrophils was measured before and after $\mathrm{LTB}_{4}$ treatment. 20 neutrophils each from three mice were randomly selected in the CFP images and examined for migration velocity during 5-min time-lapse imaging. Dots and bars indicate the migration velocity of each neutrophil and the mean values, respectively. ${ }^{* *}, P<0.01 ;{ }^{* * *}, P<0.001$ (Mann-Whitney $U$ test). (D) FRET and CFP images in Eisuke mice. The bottom left panel shows a schematic view of this image. Cr, crypt; Ly, lymphatic vessel; Ve, venule. At time 0 during time-lapse imaging, LY293111, an LTB 4 receptor antagonist, was injected intravenously at $4 \mathrm{mg} / \mathrm{kg}$. Gamma, 1.7. Image is a representative view field of a mouse in three independent experiments. Bars: (A) $50 \mu \mathrm{m} ;$ (D) $30 \mu \mathrm{m}$. (E) Time courses of the ERK activity of intravascular and interstitial neutrophils plotted against time. 10 neutrophils in each of three mice were randomly selected in the CFP images and examined for ERK activity in the corresponding FRET/CFP ratio image. Error bars indicate the one SD. (F) Neutrophils on the endothelial cells were classified into the four steps of the recruitment cascade before and after LY293111 treatment. Before and after LY293111 injection, 428 and 386 neutrophils, respectively, were scored in three mice. Error bars indicate SD. ${ }^{*}, P<0.05 ;{ }^{* *}, P<0.01$ (Student's $t$ test). (G) Effect of LY293111 on the migration velocity of interstitial neutrophils. 20 neutrophils in each of three mice were randomly chosen in the CFP images at -20 and 20 min, and the migration velocity of each neutrophil was measured. Red bars indicate mean values. ${ }^{* *}, \mathrm{P}<0.001$ (Mann-Whitney $U$ test). (H) Schematic view of the regulation of neutrophil migration. ERK plays a central role in controlling cell migration, whereas PKA regulates cell migration via ERK regulation. 
ERK through a PI3K-dependent pathway in HEK 293 cells ectopically expressing EP2 and EP4 (Fujino et al., 2003). In another study, EP4 has been shown to activate ERK in rat calvaria cell cultures treated with a specific agonist (Minamizaki et al., 2009). Unexpectedly, we found that an EP4 agonist activates PKA and suppresses ERK activity in neutrophils. The reason for this discrepancy between the previous in vitro studies and the present in vivo study remains unknown. Additional signals that could be engaged only in vivo may facilitate signaling from EP4 to $\mathrm{G}_{\mathrm{s}}$. Considering the observation with EP4 knockout mice that EP4 down-regulates inflammation (Kabashima et al., 2002), we suggest that EP4 activates PKA, inhibits ERK, and thereby suppresses neutrophil activities. Last but not least, in the in vivo experimental setup, we should keep in mind that the effect of perturbation might be indirect. For example, $\mathrm{PGE}_{2}$-mediated suppression of neutrophil activities might be mediated by cytokines secreted by $\mathrm{PGE}_{2}$-stimulated cells other than neutrophils.

It has been reported that PKA activation of neutrophils by cAMP-elevating agents blocks selectin-dependent rolling over and subsequent firm adhesion to endothelial cells (Berends et al., 1997). Another study has indicated that elevation of cAMP levels reduces neutrophil adhesion to the endothelial cells by blocking the surface expression of $\alpha \mathrm{M} \beta 2$ integrin (Derian et al., 1995). Based on these observations, it has been suggested that intracellular levels of cAMP may decrease upon neutrophil adhesion (Lorenowicz et al., 2007). We confirmed that intravenous injection of dbcAMP activated PKA in the neutrophils and decreased the number of neutrophils on the endothelial cells (Fig. 4). However, in contrast to our expectation, we found that PKA activity in the neutrophils was increased gradually during adhesion to the endothelial cells (Fig. 3). Thus, it appears that the slight increase in PKA activity does not interfere with the neutrophil recruitment to the endothelial cells. Another cAMP target, Epac, may play a critical role in the initial step of neutrophil adhesion (Lorenowicz et al., 2007). Because PKA also regulates inflammation by promoting endothelial barrier integrity (Lorenowicz et al., 2007), it would be interesting to examine the activity change of PKA in the endothelial cells. For this purpose, we need to improve the spatial resolution of FRET images in the future.

In conclusion, against current models showing that $\mathrm{PGE}_{2}$ binding to EP4 activates ERK, we found that EP4 engagement activates PKA, suppresses ERK, and thereby inhibits the neutrophil recruitment cascade, suggesting that NSAID enteritis may be caused at least partially by the enhanced neutrophil recruitment. Considering the large number of cytokines and growth factors that could regulate neutrophils or other inflammatory cells in vivo, live imaging of the ERK and PKA activities will help in deciphering the role of each cytokine in the physiological context.

\section{MATERIALS AND METHODS}

Transgenic mice expressing FRET biosensors. The generation of transgenic mice expressing the ERK FRET biosensor EKAREVnes (Eisuke mice), the PKA FRET biosensor AKAR3EVnes (PKAchu mice), and the negative control FRET biosensor AKAR3EV-NC was reported previously
(Kamioka et al., 2012). Founder animals were backcrossed more than five generations to $\mathrm{C} 57 \mathrm{BL} / 6 \mathrm{~N} \mathrm{Jcl}$. Mice were housed in a specific pathogenfree facility and received a routine chow diet and water ad libitum. To date, no disease or anomaly has been associated with the expression of the FRET biosensors used in this study. 6-16-wk-old mice were used for the in vivo imaging. The animal protocols were reviewed and approved by the Animal Care and Use Committee of Kyoto University Graduate School of Medicine (No. 10584).

Microscopy and image processing. 2PM was performed with an FV1000MVE inverted microscope (Olympus) equipped with a 30×/1.05 NA silicon oil-immersion objective lens (UPLSAPO 30xS; Olympus) and a Mai Tai DeepSee HP Ti:sapphire Laser (Spectra Physics) or with an FV1000MVE inverted microscope equipped with a $30 \times / 1.05$ NA silicon oil-immersion objective lens and an InSight DeepSee Laser (Spectra Physics). The laser power used for observation was between 4 and $8 \%$. Scan speed was set between 12.5 and $20 \mu$ s/pixel. Images were recorded every 10-30 s for long-term imaging or every $1.5 \mathrm{~s}$ for short-term imaging. The excitation wavelength for CFP was $840 \mathrm{~nm}$. We used an IR-cut filter, BA685RIF-3, two dichroic mirrors, DM505 and DM570, and three emission filters, BA460-500 (Olympus) for CFP, BA520-560 (Olympus) for YFP, and 645/60 (Chroma Technology Corp.) for Qtracker 655.

Acquired images were analyzed with MetaMorph software (Universal Imaging) as described previously (Aoki and Matsuda, 2009; Kamioka et al., 2012). In brief, the level of FRET was represented by the FRET/CFP ratio image in intensity modulated display mode; eight colors from red to blue are used to represent the FRET/CFP ratio, and the 32 grades of color intensity are used to represent the signal intensity of the CFP image. The warm and cold colors indicate high and low FRET levels, respectively. Neutrophils were distinguished from the other cell types by their characteristic segmented nuclei.

Neutrophil recruitment and extravasation were divided arbitrarily into the following five steps: rolling, adhesion, crawling, transmigration, and chemotaxis in the interstitial tissue. The term "rolling" was used in the case of round-shape neutrophils that rolled over the endothelial cells by the frame by frame comparison. Under the current scanning conditions, cells flowing in the venules could not be imaged clearly and were excluded from the designation of rolling neutrophils. "Adhesion" was the term used for neutrophils that had ceased rolling and remained in the same position for at least $30 \mathrm{~s}$. "Crawling" was used in the case of neutrophils that adopted an amoeboid shape and crawled over the endothelial cells. "Transmigration" was defined as neutrophils invading into or between endothelial cells to emigrate. Finally, the term "chemotaxis" was applied when neutrophils migrated in the interstitial tissue. Under our experimental conditions, we could not separate stochastic migration and chemotactic migration, so we referred to both as "chemotaxis."

The flux of rolling neutrophils and the number of adherent neutrophils were quantified as described previously (Kubes et al., 2003) with time-lapse images acquired every $1.5 \mathrm{~s}$. The number of rolling neutrophils (flux) was counted using frame by frame analysis for $10 \mathrm{~min}$. A neutrophil was defined as adherent to endothelial cells if it remained stationary for $>30 \mathrm{~s}$. The numbers of adherent, crawling, or transmigrating neutrophils were averaged per 400-700 $\mu \mathrm{m}$ endothelial cells during a 10-min observation period.

In vivo observation of the small intestine. Mice were anesthetized with $1.5-2 \%$ isoflurane (Abbott) inhalation and placed in the supine position on an electric heat pad maintained at $37^{\circ} \mathrm{C}$. Before surgery, the abdominal area of the mouse was disinfected using $70 \%$ ethanol. A small vertical incision was made in the right side of the abdominal wall. The small intestine was pulled out of the abdominal cavity, and both proximal and distal sides of the small intestine of interest were ligated using 5-0 silk surgical sutures (Nesco Suture). With a 29-gauge needle, $1 \mu \mathrm{g} / \mathrm{ml}$ LPS (Sigma-Aldrich) and $100 \mathrm{nM}$ fMLP (Sigma-Aldrich) were administered into the intestinal cavity. The small intestine was returned to the abdominal cavity before closing the wound with 6-0 silk sutures (Ethicon). After $2 \mathrm{~h}$, the abdominal cavity was reopened to pull out the small intestine on a cover-glass placed in a heat-stage maintained at $37^{\circ} \mathrm{C}$. The small intestine was fixed with surgical sutures to minimize 
peristalsis and observed by $2 \mathrm{PM}$. Time-lapse imaging was performed with a two-photon microscope running FluoView software (Olympus). To observe extravasation of neutrophils, we observed postcapillary venules with a diameter of $\sim 30-50 \mu \mathrm{m}$. In some experiments, $5 \mathrm{mg} / \mathrm{kg}$ PD0325901 MEK inhibitor (EMD Millipore) in PBS, $0.3 \mathrm{~g} / \mathrm{kg}$ dbcAMP (EMD Millipore), $7.5 \mathrm{mg} / \mathrm{kg}$ flurbiprofen axetil (KAKEN Pharmaceutical), $15 \mathrm{mg} / \mathrm{kg}$ SB203580 p38 inhibitor (Cayman Chemical), $7.5 \mu \mathrm{g} / \mathrm{kg} \mathrm{LTB}$ (Cayman Chemical), $4 \mathrm{mg} / \mathrm{kg} \mathrm{LY} 293111 \mathrm{LTB}_{4}$ inhibitor (Cayman Chemical), $0.5 \mathrm{mg} / \mathrm{kg}$ ONO-AE-1-259-01, $0.25 \mathrm{mg} / \mathrm{kg}$ ONO-AE1-329, or $5 \mathrm{mg} / \mathrm{kg}$ ONOAE3-208 (Ono Pharmaceutical Co.) was administered via the jugular vein during the course of in vivo imaging. The plasma half-life of ONO-AE3-208 measured in an experiment of intravenous injection was $0.2 \mathrm{~h}$ (Kabashima et al., 2002). The plasma half-lives of ONO-AE-1-259-01 and $0.25 \mathrm{mg} / \mathrm{kg}$ ONO-AE1-329 are not available, but a compound with similar structure is inactivated in the lung within $20 \mathrm{~min}$. The reagents were administered for $30 \mathrm{~s}$ except for $\mathrm{LTB}_{4}$, which was administered for 3-5 min. To visualize the blood vessels, $3 \mu \mathrm{l}$ Qtracker 655 (Invitrogen) was coinjected with the drugs. Mice were euthanized after the experiments.

Bone marrow transplantation. Bone marrow was harvested from the femur and tibia of 14-wk-old female PKAchu mice. Whole bone marrow nucleated cells were transplanted into 10 -wk-old C57BL/6 female mice $\left(10^{7}\right.$ cells/mouse), which had been subjected to $9.6 \mathrm{~Gy}$ irradiation. Mice were observed $7 \mathrm{wk}$ after transplantation.

NSAID enteritis. 10-wk-old C57BL/6 mice were administered subcutaneously with $7.5 \mathrm{mg} / \mathrm{kg}$ flurbiprofen axetil with or without $0.25 \mathrm{mg} / \mathrm{kg}$ ONO-AE1-329 or $5 \mathrm{mg} / \mathrm{kg}$ ONO-AE3-208 as described previously (Kabashima et al., 2002). For the mice administered with ONO-AE3-208, mice were provided with drinking water containing $37.5 \mu \mathrm{g} / \mathrm{ml} \mathrm{ONO}$ AE3-208. $24 \mathrm{~h}$ later, mice were sacrificed to count intestinal ulcers under a stereoscopic microscope.

Statistical analysis. P-values for normal distributed data were calculated with the Student's $t$ test or paired Student's $t$ test for the evaluation of statistically significant differences. Otherwise, the Mann-Whitney $U$ test was used. Data analysis was performed using Prism software (GraphPad Software). $*, \mathrm{P}<0.05 ; * *, \mathrm{P}<0.01 ; * * *, \mathrm{P}<0.001$.

Online supplemental material. Video 1 shows the activation of ERK in neutrophils during adhesion to endothelial cells of the inflamed small intestine. Video 2 shows the requirement of ERK activity during the neutrophil recruitment cascade. Video 3 shows the activation of PKA during the neutrophil recruitment cascade. Video 4 shows the PKA activity in neutrophils derived from a PKAchu mouse and transplanted into a wildtype mouse. Video 5 shows the PKA inhibition of ERK activity, recruitment to endothelial cells, and migration of neutrophils. Video 6 shows the accelerated migration and activation of ERK of neutrophils after NSAID injection. Video 7 shows the inhibition of the rolling step and the migration of neutrophils by an EP4 agonist. Video 8 shows the promoting effect of NSAID on neutrophil recruitment was lost in the presence of an EP4 agonist. Online supplemental material is available at http://www.jem.org/ cgi/content/full/jem.20132112/DC1

An EP2 agonist, ONO-AE1-259-01, an EP4 agonist, ONO-AE1-329, and an EP4 antagonist, ONO-AE3-208, were kindly provided by ONO Pharmaceutical Co. We thank K. Otani and Y. Hamasaki for assisting with the jugular vein cannulation and bone marrow leukocyte preparation, respectively, and Y. Inaoka, K. Hirano, R. Tabata, and A. Kawagishi for their technical assistance.

M. Matsuda and Y. Kamioka were supported the Platform for Dynamic Approaches to Living System and by a Grant-in-Aid for Scientific Research on the Innovative Area "Fluorescence Live Imaging" (No. 22113002) from the Ministry of Education, Culture, Sports, Science and Technology (MEXT) and by funds from the Innovative Techno-Hub for the Integrated Medical Bio-Imaging Project of the Special Coordination Funds for Promoting Science and Technology by MEXT, Japan.

The authors declare no competing financial interests.
Submitted: 6 October 2013

Accepted: 9 April 2014

\section{REFERENCES}

Alblas, J., E.J. van Corven, P.L. Hordijk, G. Milligan, and W.H. Moolenaar. 1993. Gi-mediated activation of the p21ras-mitogen-activated protein kinase pathway by alpha 2 -adrenergic receptors expressed in fibroblasts. J. Biol. Chem. 268:22235-22238.

Aoki, K., and M. Matsuda. 2009. Visualization of small GTPase activity with fluorescence resonance energy transfer-based biosensors. Nat. Protoc. 4: 1623-1631. http://dx.doi.org/10.1038/nprot.2009.175

Bengis-Garber, C., and N. Gruener. 1996. Protein kinase A downregulates the phosphorylation of $\mathrm{p} 47$ phox in human neutrophils: A possible pathway for inhibition of the respiratory burst. Cell. Signal. 8:291-296. http://dx.doi.org/10.1016/0898-6568(96)00052-6

Berends, C., B. Dijkhuizen, J.G. de Monchy, A.E. Dubois, J. Gerritsen, and H.F. Kauffman. 1997. Inhibition of PAF-induced expression of CD11b and shedding of L-selectin on human neutrophils and eosinophils by the type IV selective PDE inhibitor, rolipram. Eur. Respir. J. 10:1000-1007. http://dx.doi.org/10.1183/09031936.97.10051000

Bjarnason, I., J. Hayllar, A.J. MacPherson, and A.S. Russell. 1993. Side effects of nonsteroidal anti-inflammatory drugs on the small and large intestine in humans. Gastroenterology. 104:1832-1847.

Borregaard, N. 2010. Neutrophils, from marrow to microbes. Immunity. 33:657-670. http://dx.doi.org/10.1016/j.immuni.2010.11.011

Capodici, C., M.H. Pillinger, G. Han, M.R. Philips, and G. Weissmann. 1998. Integrin-dependent homotypic adhesion of neutrophils. Arachidonic acid activates Raf-1/Mek/Erk via a 5-lipoxygenase-dependent pathway. J. Clin. Invest. 102:165-175. http://dx.doi.org/10.1172/JCI592

Derian, C.K., R.J. Santulli, P.E. Rao, H.F. Solomon, and J.A. Barrett. 1995. Inhibition of chemotactic peptide-induced neutrophil adhesion to vascular endothelium by cAMP modulators. J. Immunol. 154:308-317.

Felder, J.B., B.I. Korelitz, R. Rajapakse, S. Schwarz, A.P. Horatagis, and G. Gleim. 2000. Effects of nonsteroidal antiinflammatory drugs on inflammatory bowel disease: a case-control study. Am. J. Gastroenterol. 95:1949-1954. http://dx.doi.org/10.1111/j.1572-0241.2000.02262.x

Fujino, H., W. Xu, and J.W. Regan. 2003. Prostaglandin $E_{2}$ induced functional expression of early growth response factor- 1 by $\mathrm{EP}_{4}$, but not $\mathrm{EP}_{2}$, prostanoid receptors via the phosphatidylinositol 3-kinase and extracellular signal-regulated kinases. J. Biol. Chem. 278:12151-12156. http:// dx.doi.org/10.1074/jbc.M212665200

Germain, R.N., E.A. Robey, and M.D. Cahalan. 2012. A decade of imaging cellular motility and interaction dynamics in the immune system. Science. 336:1676-1681. http://dx.doi.org/10.1126/science.1221063

Häfner, S., H.S. Adler, H. Mischak, P. Janosch, G. Heidecker, A. Wolfman, S. Pippig, M. Lohse, M. Ueffing, and W. Kolch. 1994. Mechanism of inhibition of Raf-1 by protein kinase A. Mol. Cell. Biol. 14:6696-6703.

Hata, A.N., and R.M. Breyer. 2004. Pharmacology and signaling of prostaglandin receptors: multiple roles in inflammation and immune modulation. Pharmacol. Ther. 103:147-166. http://dx.doi.org/10.1016/j.pharmthera .2004 .06 .003

Heit, B., S. Tavener, E. Raharjo, and P. Kubes. 2002. An intracellular signaling hierarchy determines direction of migration in opposing chemotactic gradients. J. Cell Biol. 159:91-102. http://dx.doi.org/10.1083/jcb .200202114

Howe, L.R., and C.J. Marshall. 1993. Lysophosphatidic acid stimulates mitogen-activated protein kinase activation via a G-protein-coupled pathway requiring p21 ras and p74raf-1. J. Biol. Chem. 268:20717-20720.

Kabashima, K., T. Saji, T. Murata, M. Nagamachi, T. Matsuoka, E. Segi, K. Tsuboi, Y. Sugimoto, T. Kobayashi, Y. Miyachi, et al. 2002. The prostaglandin receptor EP4 suppresses colitis, mucosal damage and CD4 cell activation in the gut. J. Clin. Invest. 109:883-893. http://dx.doi.org/ 10.1172/JCI0214459

Kalinski, P. 2012. Regulation of immune responses by prostaglandin $\mathrm{E}_{2}$ J. Immunol. 188:21-28. http://dx.doi.org/10.4049/jimmunol.1101029

Kamioka, Y., K. Sumiyama, R. Mizuno, Y. Sakai, E. Hirata, E. Kiyokawa, and M. Matsuda. 2012. Live imaging of protein kinase activities in transgenic mice expressing FRET biosensors. Cell Struct. Funct. 37:65-73. http://dx.doi.org/10.1247/csf.11045 
Kolaczkowska, E., and P. Kubes. 2013. Neutrophil recruitment and function in health and inflammation. Nat. Rev. Immunol. 13:159-175. http:// dx.doi.org/10.1038/nri3399

Kubes, P., B. Heit, G. van Marle, J.B. Johnston, D. Knight, A. Khan, and C. Power. 2003. In vivo impairment of neutrophil recruitment during lentivirus infection. J. Immunol. 171:4801-4808. http://dx.doi.org/10.4049/ jimmunol.171.9.4801

Liu, X., B. Ma, A.B. Malik, H. Tang, T. Yang, B. Sun, G. Wang, R.D. Minshall, Y. Li, Y. Zhao, et al. 2012. Bidirectional regulation of neutrophil migration by mitogen-activated protein kinases. Nat. Immunol. 13:457-464. http://dx.doi.org/10.1038/ni.2258

Lorenowicz, M.J., M. Fernandez-Borja, and P.L. Hordijk. 2007. cAMP signaling in leukocyte transendothelial migration. Arterioscler. Thromb. Vasc. Biol. 27:1014-1022. http://dx.doi.org/10.1161/ATVBAHA.106.132282

Megens, R.T.A., K. Kemmerich, J. Pyta, C. Weber, and O. Soehnlein. 2011. Intravital imaging of phagocyte recruitment. Thromb. Haemost. 105:802810. http://dx.doi.org/10.1160/TH10-11-0735

Minamizaki, T., Y.Yoshiko, K. Kozai, J.E. Aubin, and N. Maeda. 2009. EP2 and EP4 receptors differentially mediate MAPK pathways underlying anabolic actions of prostaglandin $\mathrm{E}_{2}$ on bone formation in rat calvaria cell cultures. Bone. 44:1177-1185. http://dx.doi.org/10.1016/j.bone.2009.02.010

Narumiya, S. 2009. Prostanoids and inflammation: a new concept arising from receptor knockout mice. J. Mol. Med. 87:1015-1022. http://dx.doi.org/ 10.1007/s00109-009-0500-1

Narumiya, S., Y. Sugimoto, and F. Ushikubi. 1999. Prostanoid receptors: structures, properties, and functions. Physiol. Rev. 79:1193-1226.

Nishigaki, N., M. Negishi, and A. Ichikawa. 1996. Two Gs-coupled prostaglandin E receptor subtypes, EP2 and EP4, differ in desensitization and sensitivity to the metabolic inactivation of the agonist. Mol. Pharmacol. 50:1031-1037.

Phillipson, M., and P. Kubes. 2011. The neutrophil in vascular inflammation. Nat. Med. 17:1381-1390. http://dx.doi.org/10.1038/nm.2514

Pillinger, M.H.,A.S. Feoktistov, C. Capodici, B. Solitar,J. Levy,T.T. Oei, and M.R. Philips. 1996. Mitogen-activated protein kinase in neutrophils and enucleate neutrophil cytoplasts: evidence for regulation of cell-cell adhesion.J. Biol. Chem. 271:12049-12056. http://dx.doi.org/10.1074/jbc.271.20.12049

Pillinger, M.H., C. Capodici, P. Rosenthal, N. Kheterpal, S. Hanft, M.R.Philips, and G. Weissmann. 1998. Modes of action of aspirin-like drugs: salicylates inhibit erk activation and integrin-dependent neutrophil adhesion. Proc. Natl.Acad. Sci.USA.95:14540-14545.http://dx.doi.org/10.1073/pnas 95.24.14540

Ricciotti, E., and G.A. FitzGerald. 2011. Prostaglandins and inflammation. Atterioscler. Thromb. Vasc. Biol.31:986-1000. http://dx.doi.org/10.1161/ ATVBAHA.110.207449

Sanz, M.J., and P. Kubes. 2012. Neutrophil-active chemokines in in vivo imaging of neutrophil trafficking. Eur. J. Immunol. 42:278-283. http:// dx.doi.org/10.1002/eji.201142231

Stadnyk, A.W., C. Dollard, T.B. Issekutz, and A.C. Issekutz. 2002. Neutrophil migration into indomethacin induced rat small intestinal injury is CD11a/ CD18 and CD11b/CD18 co-dependent. Gut. 50:629-635. http://dx.doi .org/10.1136/gut.50.5.629

Vossler, M.R., H. Yao, R.D. York, M.G. Pan, C.S. Rim, and P.J. Stork 1997. cAMP activates MAP kinase and Elk-1 through a B-Raf- and Rap1-dependent pathway. Cell. 89:73-82. http://dx.doi.org/10.1016/ S0092-8674(00)80184-1

Wallace, J.L. 2012. NSAID gastropathy and enteropathy: distinct pathogenesis likely necessitates distinct prevention strategies. Br. J. Pharmacol. 165:67-74. http://dx.doi.org/10.1111/j.1476-5381.2011.01509.x

Wallace, J.L., C.M. Keenan, and D.N. Granger. 1990. Gastric ulceration induced by nonsteroidal anti-inflammatory drugs is a neutrophil-dependent process. Am. J. Physiol. 259:G462-G467.

Winitz, S., M. Russell, N.X. Qian, A. Gardner, L. Dwyer, and G.L. Johnson. 1993. Involvement of Ras and $\mathrm{R}$ af in the Gi-coupled acetylcholine muscarinic $\mathrm{m} 2$ receptor activation of mitogen-activated protein (MAP) kinase kinase and MAP kinase. J. Biol. Chem. 268:19196-19199.

Yamane, H., Y. Sugimoto, S. Tanaka, and A. Ichikawa. 2000. Prostaglandin $\mathrm{E}_{2}$ receptors, $\mathrm{EP} 2$ and $\mathrm{EP} 4$, differentially modulate TNF- $\alpha$ and IL-6 production induced by lipopolysaccharide in mouse peritoneal neutrophils. Biochem. Biophys. Res. Commun. 278:224-228. http://dx.doi.org/10 $.1006 /$ bbrc. 2000.3779

Yokomizo, T., T. Izumi, K. Chang, Y. Takuwa, and T. Shimizu. 1997. A Gprotein-coupled receptor for leukotriene $\mathrm{B}_{4}$ that mediates chemotaxis Nature. 387:620-624. http://dx.doi.org/10.1038/42506

Zarbock,A.,T.L.Deem,T.L. Burcin, and K.Ley. 2007. $G \alpha_{\mathrm{i} 2}$ is required for chemokine-induced neutrophil arrest. Blood. 110:3773-3779. http://dx.do .org/10.1182/blood-2007-06-094565 